\title{
Mechanism of twin-reduced III-V epitaxy on As-modified vicinal Si(111)
}

\author{
Lars Winterfeld, ${ }^{1}$ Christian Koppka, ${ }^{2}$ Daniel Abou-Ras, ${ }^{3}$ Peter \\ Kleinschmidt, ${ }^{2}$ Oliver Supplie, ${ }^{2}$ Thomas Hannappel, ${ }^{2}$ and Erich Runge ${ }^{1}$ \\ ${ }^{1}$ Institute of Physics, Department for Theoretical Physics, \\ TU Ilmenau, Weimarer Straße 25, D-98693 Ilmenau, Germany \\ ${ }^{2}$ Institute of Physics, Department for Photovoltaics, TU Ilmenau, \\ Gustav-Kirchhoff-Straße 5, D-98693 Ilmenau, Germany \\ ${ }^{3}$ Helmholtz-Zentrum Berlin für Materialien und Energie, \\ Hahn-Meitner-Platz 1, D-14109 Berlin, Germany
}

(Dated: 2018-11-13)

\begin{abstract}
Rotational twins are fundamental defects in III-V epitaxy, in particular for the growth on nonpolar (111) surfaces. Based on density functional theory (DFT) calculations, we develop a general model for III-V nucleation on vicinal non-polar (111)-oriented substrates and focus on the important differences in the atomic step configuration of different miscut directions. We verify this model by a relevant materials system when growing GaP epilayers on As-terminated $\mathrm{Si}(111)$ : Scanning tunneling microscopy measurements reveal the formation of straight double bilayer steps after Aspassivation of the $\mathrm{Si}(111)$ surface, which persist after III-V growth, as we display when measuring the buried heterointerface with cross-sectional high-resolution transmission electron microscopy. A twin amount in the $\mathrm{GaP}$ epilayers is observed in dependence on the misorientation and our nucleation model explains the underlying mechanisms: The number of back-bonds at the step edges determines the nucleation site. Accordingly, the substrate misorientation towards $[11 \overline{2}]$ yields twin-suppression, which is in full agreement with experiment. Finally, we use DFT input for Kinetic Monte Carlo calculations to explain the formation of $\mathrm{GaP}$ rotational twins on $\mathrm{Si}(111)$ :As in order to explain their volume fraction observed by high resolution X-ray diffraction measurements. We thus derive a complete picture of the formation and suppression of rotational twins relevant for low-defect III-V-on-Si integration.
\end{abstract}

\section{INTRODUCTION}

A major challenge in optoelectronics is to combine the advantages of III-V semiconductors with the wellestablished silicon technology [1-4]. In particular, the realization of low defect densities is decisive for the resulting device performance. The heteroepitaxy on $\operatorname{Si}(111)$ is well suited to avoid antiphase disorder [5/7] and to reduce the threading dislocation density in the III-V epilayers 8 11. In addition, (111)-orientation is preferred for novel III-V nanowire (NW)-based devices [3]. Pseudomorphic III-V transition epilayers can, in general, facilitate the NW nucleation and are particularly important for vapor liquid solid growth[12 [15]; for a recent review see Ref. 16. Such planar buffer layers were implemented successfully for various III-V nanowire based structures such as GaAs[12, 17, InP 18, InAs 19 and $\mathrm{GaN}$ 20, 21. In particular, $\mathrm{GaP} / \mathrm{Si}(111)$ is a promising hetero-substrate for the integration of polar III-V nanowires on unpolar $\mathrm{Si}$ substrates due to the small lattice mismatch between silicon $(\mathrm{a}=5.43 \AA)$ and gallium phosphide $(\mathrm{a}=5.45 \AA) 22$, 23. Furthermore, a highquality GaP buffer enables the integration of further planar and optical active III-V epilayers into the device architecture [24].

Unfortunately, the formation of rotational twin domains (RTDs) has to be expected during the epitaxy on (111)-oriented substrates. These RTDs are necessarily accompanied by twin boundaries between the untwinned and twinned domains (defined relative to the underlying
Si substrate; cf. Fig. 1 and Ref. 251), which we denote as $\alpha$ and $\beta$, respectively. Especially for polar compound semiconductors, it can be assumed that incoherent (often referred to as lateral) twin boundaries have net charges 26] and hence a non-negligible impact on the optoelectronic properties 27, 28. We recently demonstrated that these twins also have a major influence on the surface morphology and on the subsequent growth of nanowires 29.

So far, only few investigations concerning the twin formation and suppression during epitaxy on (111)-oriented surfaces have been reported. In particular, the influence of different growth conditions on the twin formation and the mechanism of defect reduction are still rather unclear. Furthermore, systematic differences exits between samples grown by Molecular Beam Epitaxy (MBE) and by Metalorganic Vapour Phase Epitaxy (MOVPE). As an example, Proessdorf et al. observed a weak temperature dependence of their RTD ratio for MBE growth of $\mathrm{GaSb} / \mathrm{Si}(111)$ 30. In contrast, we have shown a significant suppression of the RTD formation in MOVPEgrown $\mathrm{GaP} / \mathrm{Si}(111)$ by reduction of the nucleation temperature [25].

Besides temperature, other factors have a significant influence on the twin formation in III-V epitaxy. For MBE growth of GaAs/Si(111), Suzuki et al. achieved a remarkable twin reduction [31 33]: They report a strong correlation between the final RTD ratio in the epilayer and the substrate pretreatment and nucleation conditions. In their case, the pre-evaporation of In on the 


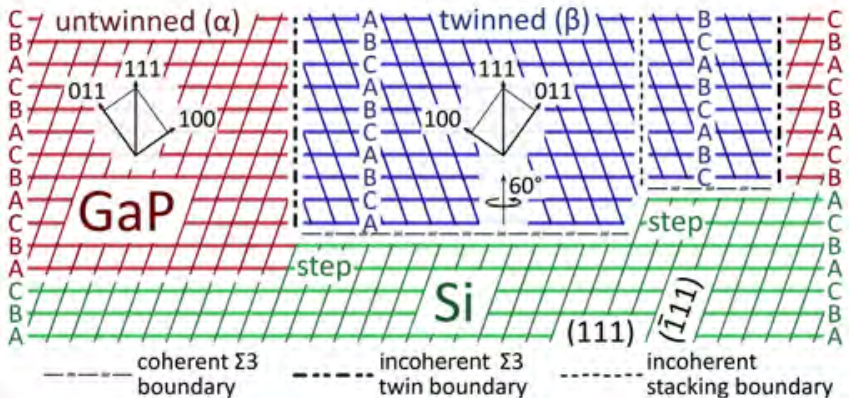

FIG. 1: Schematic cross section of GaP/Si(111):As with twin domains forming at the interface. Regular untwinned $\mathrm{GaP}$ is shown as a stacking of red (111) planes following the ABC sequence of the vicinal substrate (green planes). Twinned GaP domains are depicted as a stacking of blue planes following a CBA sequence. Three types of boundaries occur: Coherent $\Sigma 3$ boundaries are separating the twinned GaP from the substrate lattice at the interface, incoherent $\Sigma 3$ boundaries $(\alpha \mid \beta)$ are separating the twinned GaP domains from the untwinned crystallites and incoherent stacking boundaries appear between twinned domains. The latter occurs due to a stacking shift when nucleation starts at different plateaus.

$\mathrm{Si}(111)$ surface (forming small InAs islands) reduces the twin formation, while Ga pre-evaporation does not show a comparable effect. In addition, they studied the impact of the V/III flux ratio on the growth rate of GaAs 3D islands, which differs between the twinned and untwinned domains. The average nuclei size correlates with the resulting twin amount. This is in line with the improved twin suppression in the case of InAs islands (In pre-evaporation), which are much smaller compared to GaAs. For the MBE growth of GaAs and GaSb on $\mathrm{Ge}(111)$, a twin reduction was observed through the use of vicinal substrates 34. Further, the polarity in compound semiconductors seems to affect the twinning mechanism 35, 36. While homoepitaxial growth is much less affected by twin formation, twinning can be a dominating defect source during heteroepitaxy, especially on unpolar substrates [37] such as Si.

The present paper investigates based on Density Functional Theory (DFT) the mechanism that allows to reduce the twin density in III-V epilayers grown on the technologically important As-modified $\mathrm{Si}(111)$. It does this based on a detailed experimental analysis of a series of As-modified $\mathrm{GaP} / \mathrm{Si}(111)$ :As heterostructure samples (reasons for As-termination are discussed below in section IIIB]. All samples were grown and prepared under identically conditions, which are optimized for this type of system. However, they differ by the miscut angle $\varphi$ relative to the (111) direction $\left(<0.5^{\circ} \rightarrow\right.$ nominally flat, $3^{\circ}$, $\left.6^{\circ}\right)$, miscut direction $([11 \overline{2}]$ or $[\overline{11} 2])$ and by nucleation conditions during epitaxy: We varied the $\mathrm{V} / \mathrm{III}$ ratio, nucleation temperature $T_{n u c}$ and nucleation time $t_{n u c}$. The volume fraction of the twin domains in the buffer layer, $R_{\beta}$, correlates with these conditions. For quantification of $R_{\beta}$, we used high resolution X-ray diffraction (HR-
XRD). The influence of these factors can be understood in a unifying model which is developed in this work.

After quickly introducing the experimental procedure in section II] we will start by developing an atomic view of the III-V-Si(111)-interface for a flat surface in section III. In section IV] we describe the termination of the vicinal $\mathrm{Si}(111)$ surfaces with $[11 \overline{2}]$ and [112] miscut directions including their corresponding atomic step structures and show their importance on the twin formation. We continue, in section $\mathrm{V}$, to explain the experimental observations and in particular the mechanism of twin reduction in certain vicinal systems. This is achieved using a model based on element-specific DFT-energy differences between the possible incorporation sites for specific adsorbed atoms. Kinetic aspects of this model were tested and confirmed by Kinetic Monte Carlo (KMC) simulations and are presented in section VI. KMC simulations clearly reflect the influence of the substrate misorientation on the GaP nucleation including rotational twin formation, as seen in our experimental data and explained by our theoretical model.

\section{EXPERIMENTAL PROCEDURE}

The $\mathrm{GaP} / \mathrm{Si}(111)$ :As samples were prepared by MOVPE, which includes As-termination followed by the "nucleation phase" with low molar flow of Ga and P precursors and the "growth phase" with high molar flow. The samples were characterized by HR-XRD as described in Ref. 25]. A Philips CM12 electron microscope was used to record high-resolution transmission electron microscopy (TEM) images of the interface between the Asmodified, regular stepped Si substrate and the GaP epilayer. Therefore, the electron beam was aligned parallel to the [110] axis. For surface analysis, described in section IVC, the samples were transferred to our Aarhus scanning tunneling microscopy (STM) setup by an UHV-based shuttle 38 right after their preparation in MOVPE environment to avoid contaminations of the surface. In addition, X-ray photoelectron spectroscopy (XPS) measurements were performed (after STM) to confirm the absence of contaminants such as oxygen, nitrogen or carbon on the surface within the limits of detection.

\section{FLAT INTERFACES}

In heteroepitaxy, the nucleation phase is generally crucial for the crystal quality of the entire layer. The structural details (including defects) of the nucleation layer strongly affect the following growth. The strong correlation between nucleation conditions and the resulting $R_{\beta}$ observed in GaP epilayers 25] result from twinning close to the interface. Hence, we start the discussion of twin formation on vicinal substrates with a short summary of what is known about the corresponding flat interface. 
Arsenic-free $\mathrm{GaP} / \mathrm{Si}(111)$ interfaces have been studied before both experimentally and theoretically. Aspects relevant to this work will be highlighted in the paragraph III A below. Less is known about the atomic structure of As-modified GaP/Si(111):As interfaces. Therefore, we present new results from our studies in the subsequent paragraph III B.

\section{A. Flat As-free $\mathrm{GaP} / \mathrm{Si}(111)$ interface}

Typically, a thermal treatment of $\mathrm{Si}(111)$ substrates (1000 $\left.{ }^{\circ} \mathrm{C}, 30 \mathrm{~min}\right)$ under $H_{2}$-atmosphere is used in MOVPE to obtain an oxygen-free silicon surface, that is H-terminated with a $(1 \times 1)$ reconstruction. 39] In Ref. 40, we studied theoretically, which atomic structures of As-free interfaces between $\mathrm{Si}(111)$ and $\mathrm{GaP}$ are thermodynamically stable. The formation energies of such structures as a function of the chemical potential were calculated by DFT for interface models with a $(2 \times 2)$ as well as a c $(4 \times 4)$ unit cell. In this investigation, the electronic compensation at heterovalent interfaces was realized by a variation of the atomic stoichiometry within at most two layers at the interface. In case of an A-type polar GaP epilayer, which is observed for nucleation on $\mathrm{H}$ terminated $\mathrm{Si}(111)$, the abrupt Si-P interface was found to be energetically stable under P-rich nucleation conditions, while a "mixed-compensated" interface is more favorable under Ga-rich conditions. For a B-type polarity in the As-free $\mathrm{GaP} / \mathrm{Si}(111)$ system, an atomic intermixture at the interface is energetically favored and expected over the entire chemical potential range. At any temperature present in experiments, gains in entropy by intermixing are too small to outweigh the energetic differences 41.

\section{B. Flat As-modified $\mathrm{GaP} / \mathrm{Si}(111)$ interface}

Experimentally, the atomic structure of the flat Asterminated $\mathrm{Si}(111)$ surface has been investigated in detail. By cooling down the Si wafer from $\sim 800^{\circ} \mathrm{C}$ in presence of As-species, the Si top layer is replaced with As forming a stable (1x1) reconstruction [24, 42, 47].

Growing GaP on $\mathrm{Si}(111)$ :As could lead to an interface that is not atomically sharp, due to the compensation of interface charges. The element-specific atomic configuration is at this point not known experimentally. Therefore, we resort to a first-principle investigation and calculate the stability of several corresponding mixed interfaces. We consider Ga-Si-mixtures near the As layer on top of $\mathrm{Si}(111)$. In particular, we study the four selected $\mathrm{GaP} / \mathrm{Si}(111)$ :As configurations, shown in Fig. 2. In our DFT calculations, we used $\geq 12 \AA$ of vacuum above and 14 bilayers of Si below the interface. We used the PBEsol functional, which is optimized for solids. Further DFT parameters, difference between abrupt and H-terminated interfaces, etc. are given in the App. A 1 and A 2.

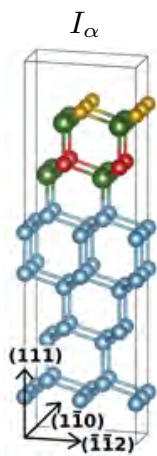

reference

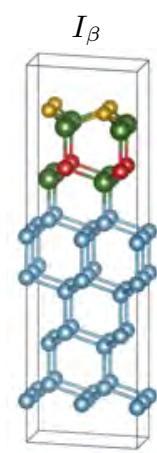

$0.05 \frac{\mathrm{eV}}{\mathrm{nm}^{2}}$

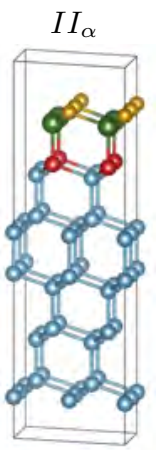

$34.89 \frac{\mathrm{eV}}{\mathrm{nm}^{2}}$

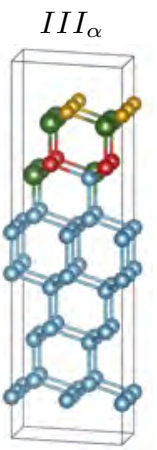

$8.50 \frac{\mathrm{eV}}{\mathrm{nm}^{2}}$

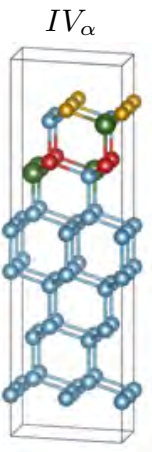

$34.74 \frac{\mathrm{eV}}{\mathrm{nm}^{2}}$
FIG. 2: Segments of the considered GaP/Si(111):As interfaces and their interface energies $\Delta \gamma_{t o t}$ (definition in main text) compared to the reference structure $I_{\alpha}$. The following color-coding is used throughout this manuscript: Si atoms are blue, As is red, Ga is green and $\mathrm{P}$ is yellow. The difference between $I_{\alpha}$ and $I_{\beta}$ is the position of the $\mathrm{P}: \alpha$ continues the normal stacking from the Si below, while $\beta$ contains $\mathrm{P}$ in cisconfiguration, starting a stacking change. For the remaining structures $I I_{\alpha}-I V_{\alpha}$ as described in the text, we only show the $\alpha$ configuration, as all $\beta$-configurations only differ in the topmost $\mathrm{P}$ positions and differences in $\Delta \gamma$ are negligible. Note that for clarity, we only show the interface region of the calculated structures. The actual unit cell contains additional vacuum, $\mathrm{Si}$ and hydrogen passivation layers.

The studied structures $I$ and $I I$ (see Fig. 2 have pristine monolayers near the As layer, while $I I I$ and $I V$ contain mixed Si-Ga layers, which are modeled with periodic $(2 \times 2)$ cells. We consider III , because it fulfills the Electron Counting Model[48, which provides a chemistryinspired heuristic guide to estimate the stability of certain atomic arrangements. Structure $I V$ has been suggested in the literature for the GaAs/Si(111) system 41. Configurations $\alpha$ and $\beta$ differ in the position of the topmost drawn $\mathrm{P}$ and the resulting stacking order, where the untwinned and twinned $\mathrm{GaP}$ domains originate, respectively. For all configurations, the difference in interface energies between $\alpha$ and $\beta$ is lower than $0.45 \frac{\mathrm{eV}}{\mathrm{nm}^{2}}$, which is comparable to stacking fault energies 49 , and is a first explanation why twins form easily. Our DFT calculations show that configuration $I$ (essentially a monolayer GaAs between $\mathrm{Si}$ and $\mathrm{GaP}$ ) is energetically preferred over the other considered interfaces by several $\frac{e V}{n^{2}}$ (numbers given in Fig. 20.

For the question of thermodynamic stability, one cannot directly compare the obtained interface energies, when the respective structures differ not only in the atomic arrangement, but also in the number of atoms per species $\left(N_{\mathrm{Si}}, N_{\mathrm{P}}, N_{\mathrm{Ga}}\right)$. In such cases, it is necessary to consider the interface energies $\Delta \gamma$ depending on the chemicals potentials, $\mu_{i}$, of the involved atomic species.

$$
\Delta \gamma A=E_{t o t}-\sum_{i} \mu_{i} N_{i}
$$

As it has been spelled out in Ref. [40, this leads in the 
present case to

$$
\Delta \gamma A=\underbrace{E_{t o t}-\mu_{\mathrm{GaP}}^{\mathrm{bulk}} N_{\mathrm{P}}-\mu_{\mathrm{Si}}^{\mathrm{bulk}} N_{\mathrm{Si}}}_{\Delta \gamma_{\text {tot }} A}-\Delta \mu_{\mathrm{P}}\left(N_{\mathrm{P}}-N_{\mathrm{Ga}}\right)
$$

where $\Delta \mu_{\mathrm{P}}=\mu_{\mathrm{P}}-\mu_{\mathrm{P}}^{\text {bulk }}$. Remembering the standard enthalpy of formation $H_{\mathrm{GaP}}^{f}=\mu_{\mathrm{GaP}}^{\mathrm{bulk}}-\mu_{\mathrm{Ga}}^{\mathrm{bulk}}-\mu_{\mathrm{P}}^{\mathrm{bulk}}$, the thermodynamically allowed range is $H_{\mathrm{GaP}}^{f} \leq \Delta \mu_{\mathrm{P}} \leq 0$. The DFT values for $\mu_{\mathrm{Si}}^{\mathrm{bulk}}=-5.747 \mathrm{eV}$ and $\mu_{\mathrm{GaP}}^{\mathrm{bulk}}=$ $-9.865 \mathrm{eV}$ (per formula unit) were calculated using the same functional and kinetic energy cutoff as the slab for a diamond and zincblende structure, respectively. We used the literature value $H_{\mathrm{GaP}}^{f}=-0.91[50]$. Within the entire range (thus both for $\mathrm{Ga}$ - and $\mathrm{P}$-rich regimes), structure $I$ remains the preferred one. We conclude that whenever Ga can exchange with Si below the As, the $\mathrm{Si}(111)$ substrate will be connected to GaP by a single GaAs layer. Since growth always happens under $\mathrm{P}$ excess in standard MOVPE based III-V growth experiments, it is useful to compare the values for $\Delta \gamma$ at $\Delta \mu_{\mathrm{P}}=0$ (the Prich regime), in which case $\Delta \gamma$ equals $\Delta \gamma_{t o t}$ (the respective values are given in Fig. 2, values for $\beta$ configurations differ by a negligible small amount).

Note that all considered configurations are of B-type polarity, which means that $\mathrm{P} \rightarrow \mathrm{Ga}$ instead of $\mathrm{Ga} \rightarrow \mathrm{P}$ bonds are realized along the [111] direction. The experimental observation that As-modification of the interface enforces this polarity 24 can be explained by a similar DFT slab calculation: The ordering Si-GaAs-GaP is energetically preferred over $\mathrm{Si}-\mathrm{GaAs}-\mathrm{PGa}$ by about $3.17 \mathrm{eV} / \mathrm{nm}^{2}$, i.e. $420 \mathrm{meV}$ per atom, which is much higher than $k_{B} T_{n u c} \approx 50 \mathrm{meV}$. Qualitatively, the same statement can be made for abrupt Si:As interfaces (like case $I I$ in Fig. 2), see App. A 2 for details.

For a better understanding of the nucleation process, we have further calculated the so-called spreading parameter $S=\gamma_{\mathrm{SiGaAs} / \mathrm{vac}}-\left(\gamma_{\mathrm{SiGaAs} / \mathrm{GaP}}+\gamma_{\mathrm{GaP} / \mathrm{vac}}\right)$ for the (111) surface from two slab calculations. We obtain $\gamma_{\mathrm{SiGaAs} / \mathrm{vac}}=19.35 \mathrm{eV} / \mathrm{nm}^{2},\left(\gamma_{\mathrm{SiGaAs} / \mathrm{GaP}}+\gamma_{\mathrm{GaP} / \mathrm{vac}}\right)=$ $21.85 \mathrm{eV} / \mathrm{nm}^{2}$ and thus $S=-2.50 \mathrm{eV} / \mathrm{nm}^{2}$. The sign $(S<0)$ indicates 3D nucleation in the initial growth phase, because $\mathrm{GaP}$ energetically prefers to create a $\mathrm{GaP}$ layer on preexisting $\mathrm{GaP}$ rather than on $\mathrm{Si}-\mathrm{GaAs}$ (structure $I$ in Fig. 2). The same is true for the abrupt $\mathrm{Si}(111)$ :As structure $I I$. In a recent study concerning GaP epitaxy on Si(111) without an intermediate buffer layer, a comparison of the surface energies suggest a Volmer-Weber growth mechanism [51, which is consistent with our results.

\section{ATOMIC STEP CONFIGURATION}

In the following, we discuss the atomic step configuration of vicinal As-terminated $\mathrm{Si}(111)$, which we show to be crucial for III-V nucleation as well as for twin formation.

\section{A. Steps on pristine $\operatorname{Si}(111)$}

Vicinal Si(111) has fundamentally different step configurations depending on the miscut direction. [52] For hydrogen-terminated pristine Si surfaces, they are known as monohydride (MH) and dihydride (DH) steps, occurring for miscuts along $[11 \overline{2}]$ and [112], respectively. These names originate from the fact that a $\mathrm{Si}$ atom at the edge of a $\mathrm{MH}(\mathrm{DH})$ step has three (two) backbonds to the Si crystal lattice, while one bond is (two bonds are) facing away from the step and saturated with hydrogen. Because we consider arsenic- instead of hydrogenterminated surfaces, we use a more general notation in this work, which is independent of termination and also valid when hydrogen is replaced with another element. Accordingly, steps after miscut along [11, $]$ are referred to as triple backbond steps (TBBS) and steps after miscut along [112] as double backbond steps (DBBS).

Note that DH steps can be further distinguished between vertical and horizontal DH steps 52. The former are, however, not stable under As treatment (due to "overhanging" As) [53. We therefore only consider the horizontal type in this work. Due to the 3-fold symmetry of the (111) surface, both step configurations possess three equivalent propagation directions $(\langle 11 \overline{2}\rangle$ and $\langle\overline{112}\rangle$ for TBBS and DBBS, respectively). Other substrate miscut directions lead to a combination of the two step configurations on the surface.

Vicinal, pristine $\mathrm{Si}(111)$ substrates with a miscut $>2^{\circ}$ react under thermal treatment different for each miscut direction [54 56]: TBBS tend to step bunching [57 59 (and thus lower step density and larger plateau width), where the number of energetically unfavorable step edges are reduced and bunched steps across several layers form another stable (111) crystal plane. For DBBS, the stability decreases with the step height and hence in most cases a higher density of single bilayer steps is realized 60 . The actual realized step configurations may depend on kinetic factors such as a time-dependent temperature gradient.

\section{B. Steps on As-terminated $\mathrm{Si}(111)$}

As-modified $\mathrm{Si}(111)$ surfaces show quite different step structures: The thermal treatment of $\mathrm{Si}(111)$ in the presence of As-species in the reactor has been shown to enforce large-scale structural rearrangements of the vicinal $\mathrm{Si}(111)$ surface and its step structure 61, 62. If the arsenic coverage exceeds $0.15-0.2$ monolayers, double bilayer steps occur for both step edge orientations. A preference for As adsorption at step edges has been suggested as possible cause of the double bilayer formation [61. This might lead to an electronically stabilized As-As configuration at the double bilayer steps.

Under sufficiently high As background pressure, the equilibrium surfaces are uniformly stepped and provide a reproducible starting condition for subsequent III-V epitaxy. If the equilibrium state during this As-passivation 
process is not reached (due to fast temperature changes, pressure ramps, etc.), also metastable structures with mixed step heights and facet formation can occur. In addition, also the presence of adatoms, or the formation of clusters or islands on the As-passivated surface was reported $53,63,64$.

Chemical passivation of the step edges is also very likely during the As-termination of the vicinal $\mathrm{Si}(111)$ surfaces 65. Such step passivation by As-termination will reduce the incorporation probability of adatoms, which may explain the prevalent formation of small islands. The presence of excess $\mathrm{Si}$ on top of the Asmonolayer stemming from a Si-As exchange process seems to be a common explanation for the source of adatoms. Antons et al. 53 performed ab initio molecular dynamics calculations showing that for vicinal Asterminated $\mathrm{Si}(111)$ TBBS are energetically favored over DBBS. In both cases As-As bonds at the step edge are more stable than $\mathrm{Si}-\mathrm{As}$ configurations. Even though these calculations were done for single bilayer steps, they strengthen the assumption of a very stable As-As step configuration, which influences the overall step formation. In other words, a complete As coverage of $\mathrm{Si}$ is plausible. Hitherto, the exact atomic structure of steps in the $\operatorname{Si}(111)$ :As system has not been experimentally verified.

\section{Experimental results}

Since the step configuration is important for nucleation, we have performed STM and HR-TEM experiments to determine their structure (see results in Fig. 3). We observe mixed step heights and facet formation for H-terminated $\mathrm{Si}(111)$ with $3^{\circ}$ miscut in $[11 \overline{2}]$, while miscut in [112] results in a smooth surface with parallel single bilayer steps (average plateau width $\sim 6 \mathrm{~nm}$ ). Both results are in line with the reported literature [57 60]. For As-modified Si(111), the STM measurements yields regular stepped surfaces for both miscut directions with an average plateau width that is consistent with double bilayer step: we measure a width of $12 \mathrm{~nm}$ matching the calculated width $\frac{2 a}{\sqrt{3} \tan \varphi}$ at the present miscut angle of $\varphi=3^{\circ}$. This STM results confirm the conclusions of Ohno et al. 61, 62, who investigated the step formation of vicinal $\mathrm{Si}(111)$ :As by LEED beam profile analysis. We emphasize that we generally do not observe step bunching at our As-terminated surfaces - even for miscut along the $[11 \overline{2}]$ direction.

Samples with miscut in $[11 \overline{2}]$ show a noticeable and reproducibly higher density of adatoms or clusters on the $\mathrm{Si}(111)$ :As surface compared to vicinal $\mathrm{Si}(111)$ in [112]. The incorporation of adatoms seems to be more reduced in case of As-passivated TBBS compared to DBBS. This is plausible since a DBBS has less atoms per area at the step edge allowing for an easier incorporation of adatoms. Compared to the reported island formation [53. 63, 64, for UHV/MBE based surface preparation,
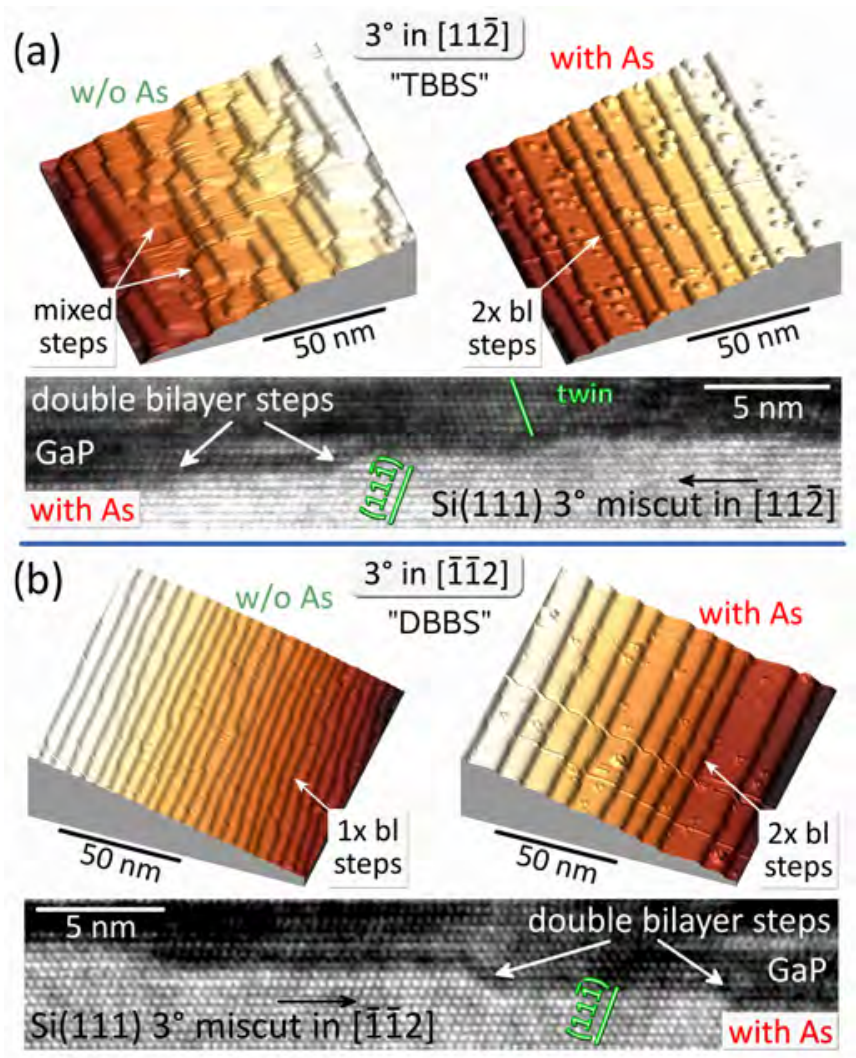

FIG. 3: Step structures of MOVPE prepared Si(111) surfaces. (a) STM measurement of As-free $\mathrm{Si}(111): \mathrm{H}$ interface with $3^{\circ}$ miscut in $[11 \overline{2}]$ shows the formation of a step-bunched surface with mixed step heights after thermal deoxidation under $\mathrm{H}_{2}$ (bias voltage: $+1.25 \mathrm{~V}$ for samples without As; $+1.44 \mathrm{~V}$ for samples with As). With thermal treatment under TBAs, a regularly stepped surface with double bilayer steps is observed. Also after GaP growth, HR-TEM measurements show the same stable, regularly arranged double bilayer steps. Here a $\mathrm{GaP}$ twin domain (lattice orientation marked in green) is observed above the step edge (TBBS type). (b) The $\mathrm{Si}(111)$ : H surface with $3^{\circ}$ miscut in [112] shows regularly arranged bilayer steps (STM), which rearrange during As-termination to double bilayer steps. HR-TEM measurements of the $\mathrm{GaP} / \mathrm{Si}(111)$ :As interface confirm the presence of double bilayer steps $(2 \mathrm{x} \mathrm{bl})$ after $\mathrm{GaP}$ growth also for this DBBS step type. Here no twin formation close to step edges is observed. For ease of comparison, all STM images have the same lateral dimensions.

our experiments reveal much smaller structures. This can be explained by a more effective desorption of adatoms in the MOVPE environment (high, constant hydrogen flow). After transfer from STM, XPS detected no contaminants on any of our samples, but only $\mathrm{Si}$ and As. While adatoms below the detection limit of XPS could still be present, it is plausible that the adatoms seen in STM are Si stemming from the Si-As exchange process during As-termination. This observation might be of interest because, to the best of our knowledge, the potentially influence of such clusters on the subsequent III-V 
nucleation has not yet been studied in the literature.

Under the experimental conditions present in our MOVPE reactor, double bilayer steps also prevail after subsequent GaP nucleation and growth, as illustrated by HR-TEM in cross-section images in Fig. 3 In case of Si(111):As with TBBS ([112] , Fig. 3(a)), twinned structures close to step edges are observed, which are not representative for $\mathrm{GaP} / \mathrm{Si}$ interfaces with DBBS (Fig. 3(b)).

\section{Impact of steps on twin formation}

If we keep all growth conditions (cf. experimental procedure in Ref. 25]) constant but only alter the miscut direction, we only change the atomic step configuration at the $\mathrm{GaP} / \mathrm{Si}(111)$ :As interface. Experimentally, this alone has a significant, reproducible effect on the macroscopic volume fraction of twins $R_{\beta}$ (cf. Fig. 4). For substrates with nominally no miscut $\left(<0.5^{\circ}\right)$ the surface step density is very low. Accordingly, the GaP nucleation mainly takes place on the flat (111) surface and is therefore significantly less affected by steps. In this case, our experiments show a high twin volume-fraction of $\sim 43 \%$, which is in line with the low energy difference between $\alpha$ and $\beta$ configurations (cf. Fig. 2). By increasing the step density (e.g., due to a substrate miscut $\geq 3^{\circ}$ ), the step configurations become more and more relevant for nucleation. In case of DBBS, a considerably suppression of RTD formation is observed. In case of TBBS, a noticeable reduction of the RTD volume fraction is visible from a substrate miscut of $<0.5^{\circ}$ to $3^{\circ}$. If the step density is further increased (substrate miscut $6^{\circ}$ ), the twin formation becomes stronger. The experimental data show that the different step types have an unequal effect on the twin formation during III-V nucleation. A reduction of the $T_{n u c}$ and $t_{n u c}$ also strongly enhances this step induced effect. The following DFT calculations should help to understand this unexpected large influence on twin formation through comparably small changes in the experimental parameters.

\section{MODEL FOR NUCLEATION AT STEPS}

\section{A. DFT results}

We took the experimental findings as starting point to build a corresponding atomic model reproducing in particular the As-terminated bilayer steps. These are summarized in the sketch Fig. 5 for both miscut directions. In our model, we build on the observation 53 that As-As bonds at the top of the step are stable edge configurations. We have therefore fixed the top edge atoms as As-As in our calculations (in principle, we do not exclude other elements in the respective atomic positions, but for the DFT calculations we are limited to a small variety of configurations). Since the exact atomic configuration of

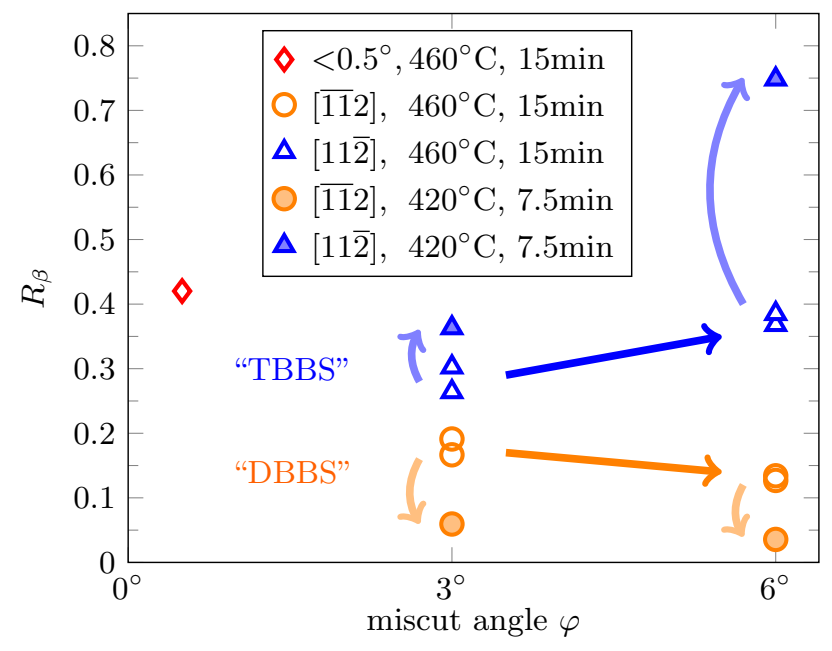

FIG. 4: HR-XRD analysis: RTD fraction $R_{\beta}$ depending on miscut angle for different nucleation temperatures and nucleation times given in the key. The red diamond marks a nominally flat surface. Orange and blue symbols mark the [112] and $[11 \overline{2}]$ direction, respectively. The darker arrows show the influence of increased miscut angle. The lighter arrows highlight the effect of a lower nucleation temperature $T_{n u c}$ combined with shorter nucleation time $t_{n u c}$.

the experimentally observed double bilayer steps is unknown, we have calculated several atomic variations concerning the lower edge atom (marked by crossed, purple atoms in Fig. 5). In particular, we performed calculations for $\mathrm{Si}$, As and $\mathrm{Ga}$ at this position. Bearing in mind our DFT results for the $\mathrm{GaP} / \mathrm{Si}(111)$ :As interface (cf. Fig. 2), we consider now both the abrupt interface and the case of a Ga layer below the As. It is conceivable, but controversial, that an energetically driven exchange process with $\mathrm{Ga}$ takes place before a noticeable $\mathrm{GaP}$ nucleation. Nevertheless, to consider this aspect and to reveal potential influences on our model, we also calculated binding energies for $\mathrm{Ga} / \mathrm{Si}$ modifications in the layer below As (marked by grey atoms in Fig. 5). Details and energetic differences are given in the App. A3.

Strikingly, there is one result common to all atomic configurations concerning the binding energy of a phosphorus (P) atom to the bilayer step: It is always energetically favored (by $0.4 \mathrm{eV}$ to $1.2 \mathrm{eV}$ per atom) that $\mathrm{P}$ takes a position where it binds to two step atoms rather than to just one. This seemingly trivial observation has the consequence that a $\mathrm{P}$ atom at a DBBS will continue the underlying Si lattice and thus nucleate in an $\alpha$ position, while a $\mathrm{P}$ atom at a TBBS has its energetic minimum in a $\beta$ position and is able to start a twinned $\mathrm{GaP}$ nuclei: This already explains why, ceteris paribus, a substrate

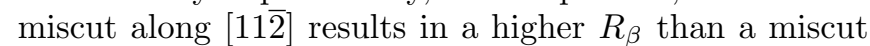
along [112] - see Fig. 4 . 


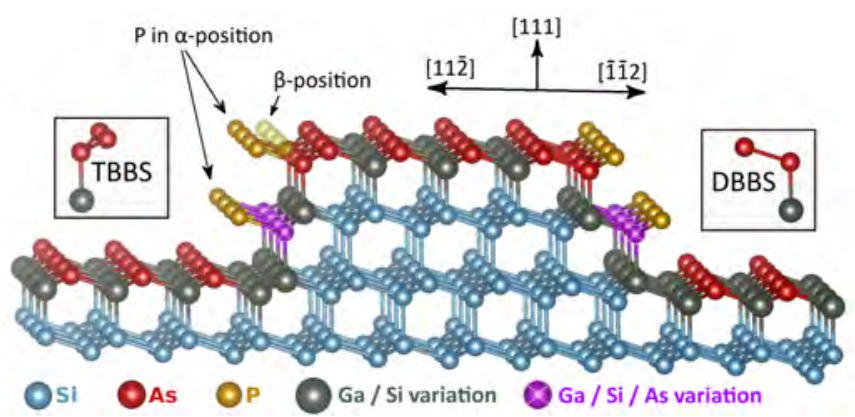

FIG. 5: Fundamental atomic structure of As-terminated $\mathrm{Si}(111)$ with double bilayer step configuration in both crystallographic directions (TBBS related to $[11 \overline{2}]$, DBBS to $[\overline{11} 2]$ ). For DFT calculations of the GaP nucleation a large number of variations of atomic configurations was used (see App. A 3

\section{B. Likely nucleation order}

The model we propose to predict likely outcomes of nucleation at steps is based on the following observations:

1. $\mathrm{P}$ adsorption is generally more likely than Ga adsorption, as long as growth happens under a large excess of $\mathrm{P}$ ( $\mathrm{Ga}$ adsorption rate limits the overall growth rate). We calculated (see App. A3) that this is even true at step edges, also in the case of As as edge atom.

2. If nucleation starts at a step (generally favored over plateau sites), $\mathrm{P}$ adsorption and diffusion is more likely to end at a position where $\mathrm{P}$ is bond to two atoms rather than just one (our DFT results).

3. While atoms in bulk III-V material forms four bonds, the group- $\mathrm{V}$ elements $\mathrm{As}$ and $\mathrm{P}$ can generally form up to five bonds.

4. Ga binds to P more likely than to As (known, e.g., from calculated interface energies).

We also assume that despite some surface stress, the displacement of the adatoms is small enough so that they can still be assigned to their lattice positions. We have confirmed this with DFT calculations (allowing for lattice relaxation) and remark that this is plausible due to the small lattice mismatch between $\mathrm{Si}$ and $\mathrm{GaP}$.

We shall argue now that these rules suggest a specific path of nucleation events. A subsequent internal reorganization of the lattice is, of course, possible, but less likely after additional layers have grown on top, because such a reorganization would require a collective movement of many atoms. For clarity, we describe in detail one particular path on As-terminated Si with double-bilayer steps as starting point. Note that the general argument on how TBBS has a tendency to form $\beta$ configurations is also valid for different step heights and edge atoms $(\mathrm{Si}$, Ga and even As, see App. A 3. The illustrated configurations in Fig. 6]+5only represent our DFT calculations.
We do not exclude several additional atomic variations at the steps.

(a) twin nucleation at $\bigcirc$ si $\bigcirc \mathrm{As} \bigcirc \mathrm{P} \odot \mathrm{Ga} \odot \mathrm{Ga} / \mathrm{Si}$ variation

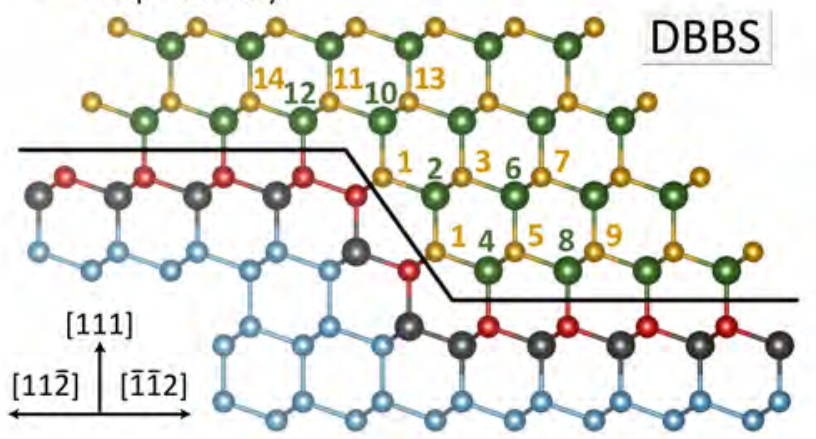

(b)

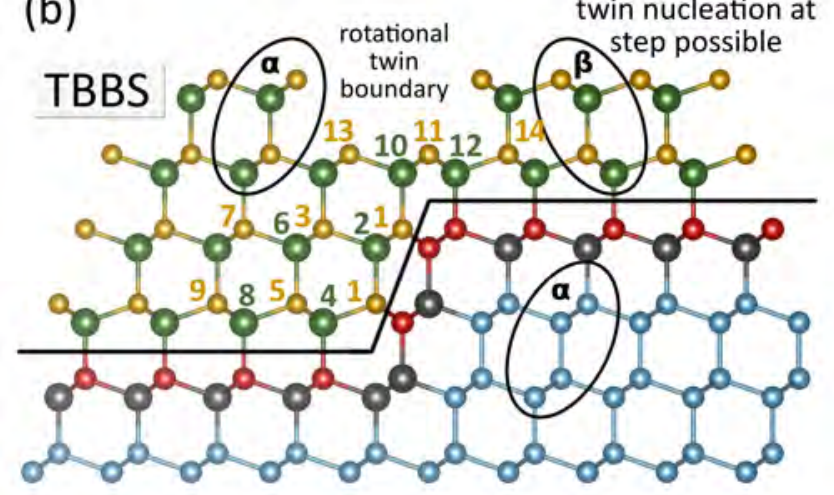

FIG. 6: Outcome of likely GaP nucleation scenarios: (a) Nucleation at a double bilayer step with DBBS configuration. At DBBS, only $\alpha$-GaP nuclei form. Hence, twin formation is only possible on a plateau. (b) Nucleation scenario at a double bilayer step with TBBS configuration. TBBS enforces $\alpha$ GaP "below" and potentially $\beta$-GaP "above" the step. Hence, twin formation is possible on plateau as well as on upper step edges).

At DBBS, this model predicts the nucleation of an $\alpha$ domain both below and above the step. For an illustration, see Fig. 6(a). Note that bonds appearing "short" in projection actually correspond to two bonds, which are inclined relative to the drawing plane. Bonds appearing "long" are parallel to the drawing plane. Everything below the black line is assumed to already exist before the nucleation of $\mathrm{GaP}$ on top. According to the above model, a new $\mathrm{P}$ atom (labeled "1" in Fig. 6(a)) will bind at the edge of the step and connect to two As atoms, where its binding energy is between $0.47 \mathrm{eV}$ and $3.00 \mathrm{eV}$ more advantageous than on the As-terminated plateau. The values depend on the atomic step configuration, different variants are listed in the App. A3. Next, Ga atoms will be bond (labeled " 2 " in Fig. 6(a)) and the next P adatoms ("3") will then take a position where there are bonds to two Ga atoms (as can be seen by "short" Ga-P bonds). By this process, the Si lattice of the substrate is 
continued into GaP, thus DBBS enforces the nucleation of an $\alpha$-domain (both below and above the step). This explains the decreasing $R_{\beta}$ with increasing miscut angle $\left(0^{\circ} \rightarrow 3^{\circ} \rightarrow 6^{\circ}\right.$ in Fig. 4 4$)$ in the experimental data along [112] as this is accompanied by an increased DBBS density, which decreases the plateau width and thereby the twin nucleation possibility.

At TBBS, the situation is different and more complex, see Fig. 6(b). P adatoms (after adsorption and diffusion) also bind to two As atoms at the step (labeled "1" in Fig. 6(b) with "short" As-P bonds). The key difference to DBBS is that for TBBS, this corresponds to a $\beta$ position that does not continue the substrate's lattice orientation. Subsequently, the Ga bonds at position "2", where it is connected to three $\mathrm{P}$ atoms, which only allow for the next $\mathrm{P}$ atom to bind at position " 3 ". Nucleation will then continue sidewards by Ga at position "4" (predetermined through the As below), $\mathrm{P}$ atoms at positions "5" binding to two Ga atoms, a new Ga at "6" binding to three atoms, and so forth. We have marked the process of growing sidewards until "9". Eventually, Ga and P will absorb above the TBBS (or diffuse there) and finally bind at positions "10", " 11 " and " 12 ", where the $\mathrm{P}$ at " 11 " has four bonds to the Ga below. Since "10" and "12" already have three bonds, the next $\mathrm{P}$ atoms binding to them ("13" and "14") can only be in the shown positions (connected by "long" bonds). In this way, an $\alpha$-domain will grow from below TBBS (in 6(b) on the left of the step edge), while a $\beta$-domain is allowed to form above the step (in 6(b) on the right of the step edge). When the $\alpha$ and $\beta$ nuclei grow, they will eventually result in an incoherent $\alpha \mid \beta$ twin domain boundary. By atomic reorganization, this boundary can shift laterally and, e.g., expand the $\beta$ domain and thereby form an additional coherent twin boundary parallel to the (111) plane - as it is visible in our HR-TEM image (3(a)). Note that nucleation below the step is energetically preferred, so we can expect TBBS to create a tendency towards more $\alpha$ than $\beta$, i.e. with lower $R_{\beta}$ than on a flat plateau. Indeed, in Fig. 4 we see a reduced $R_{\beta}$ for $3^{\circ}$ miscut along [11 $\left.\overline{2}\right]$ compared to the flat substrate.

The above argument holds as long as nucleation starts with $\mathrm{P}$ adatoms binding at the TBBS. If, on the other hand, there already is a $\mathrm{GaP}$ lattice growing on the lower level near the TBBS, then a mobile $\mathrm{P}$ atom in that region gains more energy by continuing that $\mathrm{GaP}$ lattice. This situation may arise, when, e.g., nucleation starts on the plateau or when the $\beta$-domain above one TBBS grows towards the next TBBS edge further above. The latter process is more likely if the plateau width is smaller, i.e. we can expect a high $R_{\beta}$ for larger miscut angles along $[11 \overline{2}]$. Indeed, Fig. 4 shows this trend for $6^{\circ}$ miscut along $[11 \overline{2}]$.

Based on our experimental findings, we have detailed our nucleation model for double bilayer steps. Still, the general preference of $\alpha$ and $\beta$ at DBBS and TBBS is also valid for single bilayer steps: Starting with a configuration analogously to Fig. 6, but with single bilayer steps, the above arguments can be repeated almost verbatim, because TBBS prefer $\beta$ configuration in any case.

As pointed out above, a complete As-termination is plausible and was assumed for the calculations. Considering that the P-Si binding energy can be higher than the one of P-As (see App. A 3), one could in principle imagine an exchange reaction at the step of $\mathrm{Si}$ and the As atom adjacent to $\mathrm{P}$ atom "1". Such a Si atom would only cause an even stronger bond and not change the order of subsequent adsorption (of Ga atoms "2" and "4", etc.).

As intermediate summary, we have established a qualitative explanation for the influence of miscut direction and miscut angle. The described nucleation scenario in detail might just be one out of many, but it agrees very well with our experimental findings and the basic mechanism (TBBS: twin formation allowed, due to the energetically favored $\beta$-position; DBBS: twin formation at step edge not viable) consistently describes many aspects and variations.

\section{KINETIC NUCLEATION MODEL}

In this section, we extend our modeling and propose an explanation for the influence of nucleation time and temperature. We first remark on the connection between nucleation patterns and volumetric data $\left(R_{\beta}\right)$.

\section{A. From nucleation to volume data}

Using DFT, we calculated the interface energy between $\mathrm{GaP} \alpha$ and $\beta$-domains. Whenever $\alpha$ grows on top of a $\beta$ domain in [111]-direction (and vice versa), this stacking change creates a coherent $\Sigma 3$ interface 66] with an energy of only $\gamma_{\text {coh }}=0.10 \mathrm{eV} / \mathrm{nm}^{2}$. On the other hand, an incoherent $\Sigma 3$ twin boundary between $\alpha$ and $\beta$-domains requires a much larger energy between $\gamma_{\text {inc }} \approx 7.1 \mathrm{eV} / \mathrm{nm}^{2}$ and $\gamma_{\mathrm{inc}} \approx 8.4 \mathrm{eV} / \mathrm{nm}^{2}$ according to DFT calculations. The value mainly depends on the exact crystallographic direction of this incoherent boundary. An $\alpha$-domain can grow over a nucleus in $\beta$ configuration (and vice versa), thereby eliminating the grain boundary along the circumference $p$ of the $\beta$-nucleus. However, this requires energy $\gamma_{\mathrm{coh}} A$ proportional to the lateral area $A$ of the nucleus for a stacking change. Since the area $A=b r^{2}$ grows one power faster than the circumference $p=c r$ (for some geometric constants $b$ and $c$ ), we can expect from energetic considerations that small nuclei will be "healed out" using this mechanism, while domains larger than about a critical size $r^{*}=\frac{h c \gamma_{\text {inc }}}{b \gamma_{\text {coh }}}$ will prevail and continue with $\alpha \mid \beta$ grain boundaries to the $\mathrm{GaP}$ surface (cf. Fig. 5 and 6 in Ref. 25]). For a bilayer height of $h=\frac{a}{\sqrt{3}}=3.2 \AA$ and nuclei with shape of an equilateral triangle, this suggests stability for nuclei with $A^{*} \approx 35 \AA^{2}$ or larger. This high energy $\gamma_{\text {inc }}$ is also a major reason for the formation of few nuclei with larger area (Fig. 8), because many 
small nuclei would cause a higher amount of incoherent $\alpha \mid \beta$ interface. In other words, $\alpha$ and $\beta$ domains have the tendency to stay separated (although they consist of the same III-V material).

Already for these reasons, it is important to consider not only the fraction of atoms in $\beta$-configuration on the substrate, but also the nuclei shapes and size distributions: While one $\beta$ nucleus with a large area $\mathrm{A}$ is likely to continue to grow up to the surface, several small nuclei adding up to the same total area A can be easily overgrown (and thus healed out). A detailed analysis of the connection from the GaP structures close to the $\mathrm{GaP} / \mathrm{Si}$ interface to defect planes and twin propagation in the GaP volume is beyond the scope of this paper. We will therefore not attempt a quantitative exact model for $R_{\beta}$ data, but rather explain tendencies and functional dependencies in the next sections.

\section{B. Interplay of temperature and V/III ratio}

Two important aspects of a varied V/III ratio can be derived from results reported in the literature: (i) According to the investigations of Stringfellow et al. 67], the thermal decomposition of the phosphorous precursor (TBP) reduces significantly below $T_{n u c}=500^{\circ} \mathrm{C}$, thus reducing the availability of phosphorous. Below $T_{n u c}=400^{\circ} \mathrm{C}$, no noticeable GaP nucleation (3D nuclei; cf. Fig. 8 below) is observed in our SEM measurements. We indirectly observe this missing nucleation also in our HR-XRD data: In Fig. 7, we plot the volume fraction of the twin domain in the buffer layer, $R_{\beta}$, depending on $T_{n u c}$ for our samples. In agreement with the reports by Stringfellow, we find a sudden increase of the twin amount at $T_{n u c}=400^{\circ} \mathrm{C}$. White light interferometry and $\mathrm{X}$-ray reflectivity measurements reveal a $\sim 11 \mathrm{~nm}$ thinner GaP epilayer compared to the other samples and thereby confirm impeded nucleation. (ii) According to the work of Suzuki et al. (MBE of GaAs on $\mathrm{Si}(111)$ ) 31, the twin amount decreases with increasing $\mathrm{V} / \mathrm{III}$ ratio, which can be confirmed by our experiments (cf. Fig. 11 in the appendix). Looking at this effect alone, one might first expect that lower $T_{n u c}$ reduces the $\mathrm{P}$-supply and thereby counteracts the suppression of twin formation. This is contrary to our XRD data and thus Fig. 7 cannot be explained alone by a reduction of the effective $\mathrm{V} / \mathrm{III}$ ratio with decreasing nucleation temperature. Thus, $T_{n u c}$ must have an additional effect on the growth of the GaP transition layer.

\section{Nucleation temperature}

Fig. 7 shows an Arrhenius-type dependence of $R_{\beta} \propto$ $\exp \left[-\frac{E_{A}}{k_{B} T_{n u c}}\right]$ on $T_{n u c}$ above $T_{n u c} \approx 400^{\circ} \mathrm{C}$. This is remarkable because both canonical thermodynamics and classical nucleation theory would predict a different

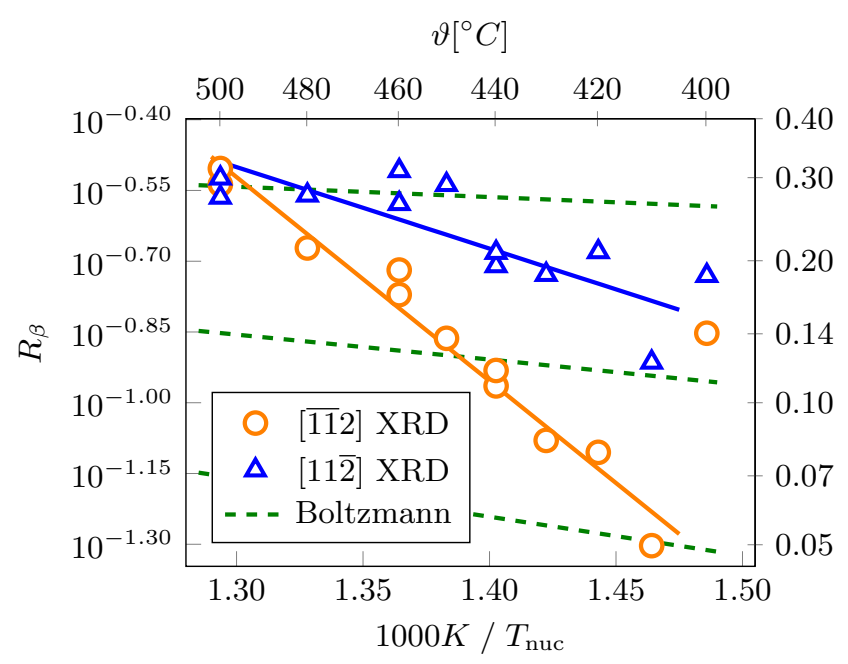

FIG. 7: Our HR-XRD data 25 in an Arrhenius-like plot of the RTD fraction $R_{\beta}$ in the GaP layer depending on the nucleation temperature for the two miscut directions [112] and $[11 \overline{2}]$ with $3^{\circ}$ miscut, $t_{n u c}=15 \mathrm{~min}$. Right and top axes labels show the value of $R_{\beta}$ and temperature in ${ }^{\circ} \mathrm{C}$ for better orientation. Dashed lines represent the Boltzmann expression A1 of Appendix A 4 for different energy differences. Solid lines show Arrhenius fits.

function dependence, $R_{\beta}=\left(1+\exp \left[-\frac{\Delta E}{k_{B} T_{n u c}}\right]\right)^{-1}$. In App. A 4, we derive this and explain why it cannot be approximated with a Boltzmann factor. Hence, those (near) equilibrium theories are not appropriate to describe our experiments and we conclude that kinetics is a dominant factor in the growth process.

Higher nucleation temperature $T_{n u c}$ causes increased dynamics of the precursors on the substrate surface. Discussing the spreading factor in section IIIB, we have shown that GaP energetically prefers to grow on preexisting $\mathrm{GaP}$ rather than on the substrate. In combination, these two observations allow to explain the island formation of GaP for different $T_{n u c}$ which we see in SEM images taken after nucleation in Fig. 8. For high $T_{n u c}=500^{\circ} \mathrm{C}$, the adsorbed $\mathrm{Ga}$ and $\mathrm{P}$ species have a large diffusion length and are thus likely to stick to a preexisting $\mathrm{GaP}$ nucleus, thereby reducing the energetically unpreferred interface to the substrate. In addition to the generally decreased nucleation density with increasing temperature, Ostwald-type ripening also suppresses the formation of new, small GaP clusters. We therefore see a large average nucleus size (diameter approximately $150 \mathrm{~nm}$ ) in this case and even after nucleation the underlying substrate can still be seen as dark areas in the background in Fig. 8 .

For low $T_{n u c}=420^{\circ} \mathrm{C}$, the diffusion length (adatom mobility) is reduced and thus, adsorbed particles are more likely to remain in the vicinity of their adsorption position. Even though the lower temperature leads to reduced TBP dissociation [67] and thereby counteracts the 


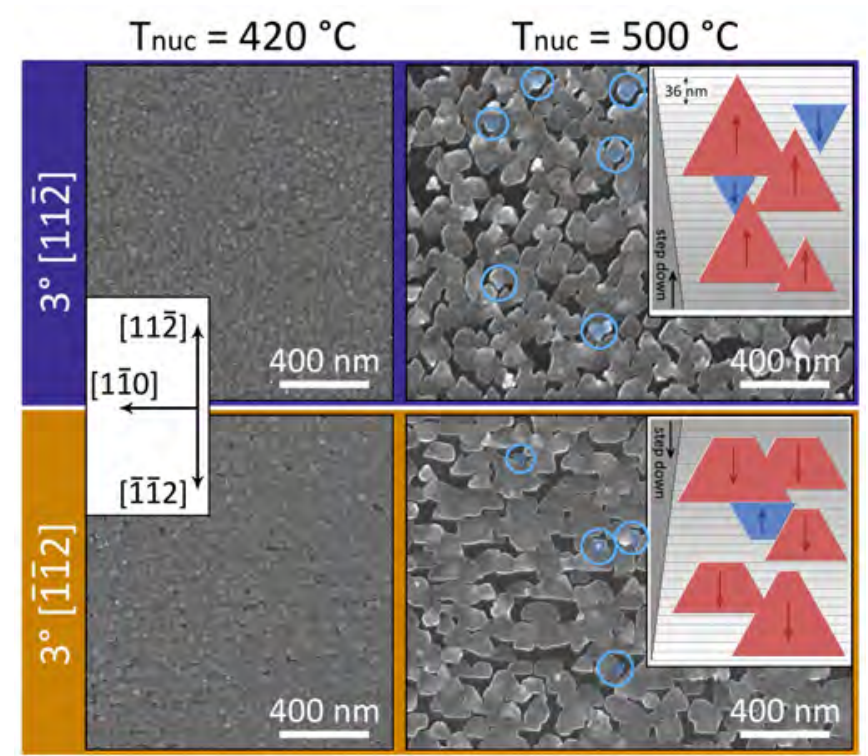

FIG. 8: SEM images of 3D GaP nuclei (SK- or VW-growth) on vicinal $\mathrm{Si}(111)$ :As. The MOVPE process was stopped directly after the nucleation step. Even an additional heat up step to the growth temperature of $T_{\text {growth }}=660{ }^{\circ} \mathrm{C}$ did not change the morphology noticeably. The average nuclei size increases with increasing $T_{n u c}$ while the surface coverage decreases (due to lower nucleation density and Ostwaldlike ripening). At $T_{n u c}=500^{\circ} \mathrm{C}$, the nuclei show a triangular shape and have overgrown in average $\sim 10$ double bilayer steps. The nuclei arrangement is influenced by the substrate steps, which leads to different morphologies for both miscut directions (visible also by comparing the darker regions in which no GaP has nucleated). The images are reminiscent of Stranski-Krastanow and Volmer-Weber growth. Blue circles highlight the nuclei orientation. All four samples were grown with $t_{n u c}=7.5 \mathrm{~min}$ and a nominal $\mathrm{V} / \mathrm{III}$ ratio $=432$.

reduction of the Ga mobility on the surface, the substrate is more uniformly covered with a high density of much smaller nuclei (average diameter approximately 30nm), which leads to an increased layer coalescence. These trends are also described in earlier work [5, 28, 68, 70. and show the reason why the use of a two-step growth approach is beneficial for III-V epitaxy on Si substrates.

In the previous subsection, we have established that small $\beta$-nuclei (which are seen for low $T_{\text {nuc }}$ ) can be healed out more easily than larger ones (which are seen for high $\left.T_{n u c}\right)$. This explains why we observe considerably larger $R_{\beta}$ with increasing $T_{n u c}$ (for both miscut directions) - see Fig. 77. Two more effects contribute to this trend: First, we can expect a higher fraction of GaP clusters on the substrate in $\beta$-configuration for increasing temperature for thermodynamic reasons. That is to say, the energetic difference $\Delta E_{\alpha \beta}$ between atoms in the preferred $\alpha$ and the $\beta$ configuration decreases relative to $k_{B} T_{n u c}$ for increasing $T_{n u c}$. Second, larger nuclei are less likely to switch between $\alpha$ and $\beta$ configuration, because this would require a collective move of many atoms. Consequently, small nuclei present at low $T_{n u c}$ are more likely to switch back to the energetically preferred $\alpha$-configuration. For these reasons, the nuclei at $T_{n u c}=420^{\circ} \mathrm{C}$ for [112] can merge easily (see Fig. 8 ) because there are only irrelevant $\alpha \mid \alpha$-boundaries, whereas many individual nuclei can be identified for $T_{n u c}=500^{\circ} \mathrm{C}$ because their merging is hindered by $\alpha \mid \beta$-boundaries. These barriers between the nuclei differentiates the nucleation phase from classical Volmer-Weber growth. After the nucleation phase, the substrate is mainly covered with a GaP nucleation layer (coverage is $T_{n u c}$ and $t_{n u c}$ dependent). In the subsequent growth phase, the temperature is raised to $660^{\circ} \mathrm{C}$ and mostly layer-by-layer growth takes place, which we conclude from the observation that the GaP surface is very smooth 25 .

Finally, we can explain two more observations concerning $R_{\beta}\left(T_{n u c}\right)$ (see Fig. 7). First, the slope is smaller for miscut along [112] . For higher $T_{n u c}$, the effective influence of the steps is reduced, because larger nuclei overgrow several plateaus and atoms initially bond to the step can more easily overcome the energy barrier to diffuse away from it. Therefore, we see a trend towards the same $R_{\beta}$ for both miscut direction for high $T_{n u c}$ and a steeper slope of $R_{\beta}$ over $T_{n u c}$ for [112] (where DBBS enforces only $\alpha$ ) as compared to [11 $\overline{2}$ ] (where TBBS enforces both $\alpha$ and $\beta$ ). Second, the mean deviation from the line of best fit is higher for [112]]. In the presence of both $\alpha$ and $\beta$ clusters (as enforced by TBBS), the realized structures and domain boundary shapes involve an additional stochastic element. This results in higher sample-dependent deviations from the general trend.

\section{Nucleation time}

The choice of the nucleation time is another influential factor for the suppression of twin formation. The rate of material flux $\dot{m}$ is the same for different $t_{n u c}$ and thus, higher $t_{n u c}$ results in more deposited material in the nucleation phase. An intentionally small $\dot{m}$ results in only approx. $11 \mathrm{~nm}$ and $21 \mathrm{~nm}$ (on average) of deposited material after $7.5 \mathrm{~min}$ and $15 \mathrm{~min}$ for nucleation at $T_{n u c}=500^{\circ} \mathrm{C}$, respectively. This leaves time for internal reorganization of the first $\mathrm{GaP}$ nuclei before they coalesce as bulk material by subsequent growth. Such reorganization allows to reduce defects. If an $\alpha$-domain from below a TBBS and a $\beta$-domain from above another TBBS meet, for instance, the boundary between them (cf. Fig. 1) can still diffuse (by rearrangement of atoms near the $\alpha \beta$ boundary).

With this understanding, we can explain the tendencies in Fig. 4. In the case of miscut along [112] (plotted in orange), lowering simultaneously $T_{n u c}$ and $t_{n u c}$ decreases the twin ratio $R_{\beta}$. We attribute this mainly to a lower $T_{n u c}$ and predict that is this case, there is only a minor influence of $t_{n u c}$ concerning the reduction of $R_{\beta}$ for the following reasons. Since most nuclei at DBBS are in $\alpha$ configuration, subsequent growth continues those 
untwinned nuclei. The nucleation time simply has to be sufficiently high to allow for the formation of nuclei covering a substantial percentage of the substrate. In practice this means a slightly higher $t_{n u c}$ is necessary with higher $T_{n u c}$ (due to the nuclei sizes) to obtain the desired layer coalescence in the subsequent growth step.

In case of miscut along [11̄] (plotted in blue in Fig. 4), the situation is different. Lowering simultaneously $T_{n u c}$ and $t_{n u c}$ increases $R_{\beta}$. Lowering $T_{n u c}$ alone would actually result in lower $R_{\beta}$; this is seen in Fig. 7 for a miscut of $3^{\circ}$ and due to the understanding developed above we can predict that the same is true for $6^{\circ}$. The profound influence of $t_{n u c}$ is that a longer nucleation time with low material influx $\dot{m}$ allows for more diffusion and reorganization of the GaP nuclei and therefore more homogeneous regions and hence, more untwinned $\alpha$ crystallites. During growth, a much higher $\dot{m}$ does not leave time for such defect-reduction processes, such that we can expect much higher $R_{\beta}$ for lower $t_{n u c}$ (ceteris paribus).

\section{E. Kinetic Monte Carlo}

As kinetics is an important aspect of nucleation, we have performed Kinetic Monte Carlo (KMC) simulations for the initial phase of GaP growth on As-terminated Si. More specifically, we chose the on-lattice, bond-counting version of KMC with periodic boundary conditions. For best performance, we chose a rejection-free implementation and used a hash-table to store the set of all possible events allowing to update this set efficiently. As events, we allow absorption, desorption and diffusion for both $\mathrm{Ga}$ and P. Hence, the state of the system is completely defined by all atom positions. The underlying lattice is of zincblende type in [111] direction, additionally allowing for both $\alpha$ and $\beta$ configurations.

We choose the adsorption probability to be the same for every possible position. That way, we model random adsorption from the gas phase both at the steps and on the terraces. Possible positions are all empty lattice positions having at least one bond to another already absorbed atom.

We also allow for desorption of previously absorbed atoms. As probability, we chose a prefactor multiplied by $\exp \left[-\Delta E / k_{B} T\right]$, where $\Delta E$ is the cohesive energy lost due to desorption. The prefactor ( $\sim$ desorption rate) is chosen such that desorption occurs rarely. Thus, more than $99.9 \%$ of the steps are diffusion events. A diffusion from a position $\overrightarrow{r_{1}}$ to $\overrightarrow{r_{2}}$ is allowed (only) if desorption from $\overrightarrow{r_{1}}$ and adsorption at $\overrightarrow{r_{2}}$ is allowed: As destination $\overrightarrow{r_{2}}$, we allow all $\alpha$ and $\beta$ positions within the same (111) layer and also within the adjacent layers below and above (27 in total). Thus, we do not restrict the system to layer-by-layer growth, but allow 3D nucleation. The Monte Carlo probability for a transition is $p_{1 \rightarrow 2}=\exp \left[-\Delta E_{12} / k_{B} T\right]$, where $\Delta E_{12}$ is in our case the difference in cohesive energy before and after the transition. To calculate the cohesive energy, we utilize
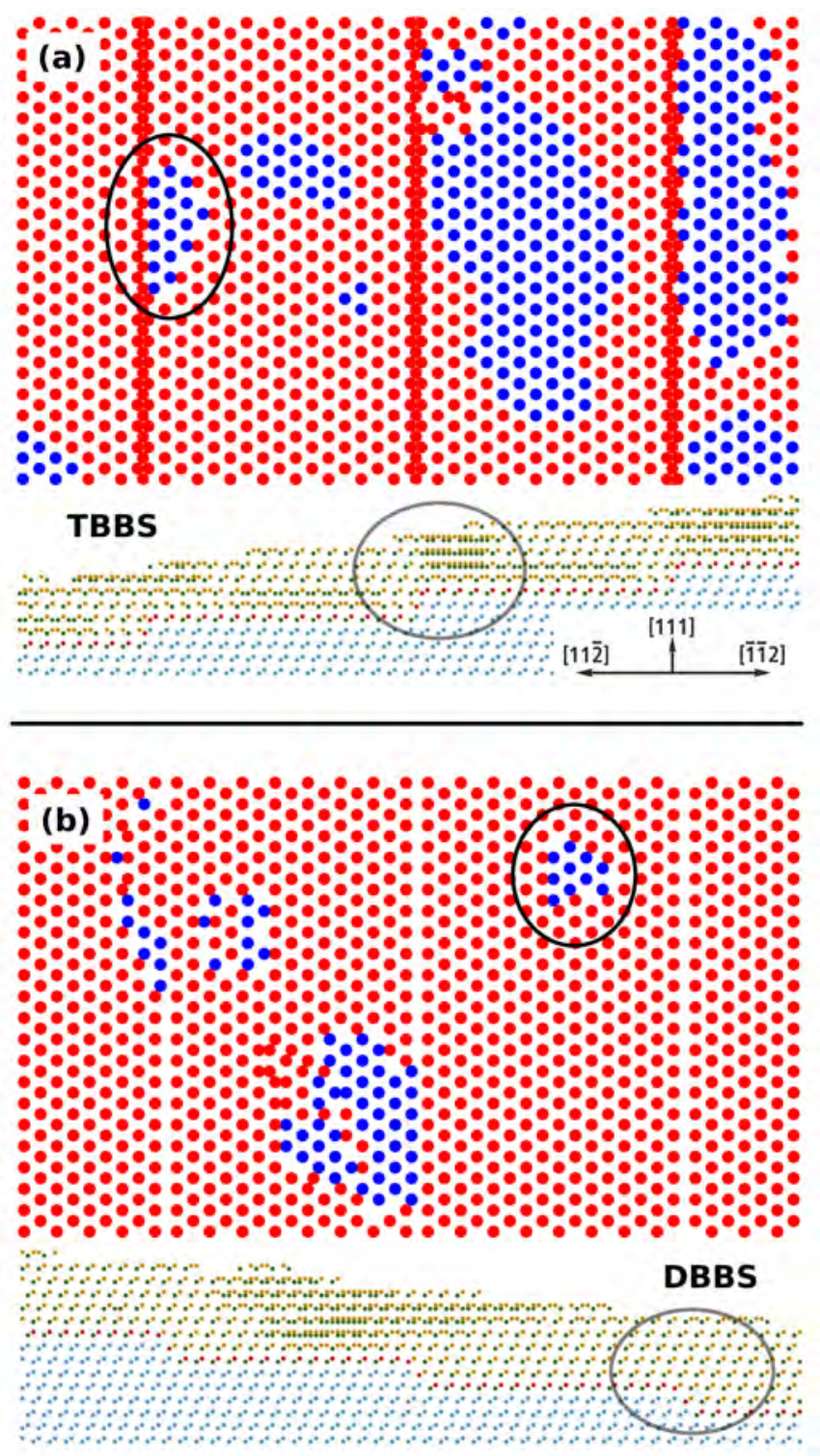

FIG. 9: Kinetic Monte Carlo results for miscut along (a) [11 $\overline{2}]$ and (b) [112]. In the upper parts, red and blue mark phosphorous atoms two bilayers above substrate in $\alpha$ and $\beta$ positions, respectively. The lower parts show a projected cross section with the same color coding as in Fig. 6 See main text for discussion.

a bond-counting model, where we incorporate bonds to nearest neighbors and in case of $\mathrm{P}$ atoms also to nextnearest neighbors. The latter is chosen, because $\mathrm{P}$ atom positions determine the growth of either $\alpha$ or $\beta$ domains (whereas Ga atoms always follow on top of $\mathrm{P}$ atoms in [111]-direction during GaP growth, compare Fig. 60). Energies for each type of bond are taken as average values over several DFT calculations for systems involving that particular bond type. After each simulation step, only events involving the surroundings of the previous event location need a recalculation of their probability. 
Fig. 9(a) and (b) show a resulting section of a KMC simulation after ten million steps for miscut along $[11 \overline{2}]$ and [112], respectively. In the upper parts of the figure, $\mathrm{P}$ atoms in red (blue) mark atoms in $\alpha(\beta)$ configuration, two bilayers above the substrate. A P atom is marked as $\alpha$ if the majority of atoms in the $\mathrm{P}$ layer below have the same relative positions as they would have in the underlying Si substrate (and $\beta$ otherwise). The lower parts show cross sections of the same simulation. Note that in this projection, atoms from different $\alpha$ and $\beta$ position can be seen apparently close to one another. Even though all parameters other than the miscut are the same for the simulations in Fig. 9(a) and (b), we can see a preference for $\alpha$ nucleation (red atoms) for miscut along [112] in 9 (b). This agrees with experiment. Further, the black ellipses highlight that $\beta$ nucleation starts often at the upper parts of steps for TBBS and may start on plateaus for both TBBS and DBBS. The temporal nucleation order and the resulting positions at DBBS and TBBS steps found by KMC are in agreement with the discussion in section VB. The grey ellipses in the cross sections highlight again that GaP can overgrow DBBS without causing additional defects, while TBBS are prone to cause twin boundaries. This is an additional affirmation for the simple model presented above.

\section{CONCLUSIONS}

By studying the interface and step configurations on an atomic scale with DFT and Kinetic Monte Carlo calculations, we were able to identify the dominant mechanism of defect-reduced III-V epitaxy on As-modified vicinal $\mathrm{Si}(111)$. Moreover, we show that the influence of $\mathrm{Si}(111)$ :As surface-step arrangements on the GaP nucleation and twin formation can be well understood from a theoretical point of view. In particular, vicinal $\mathrm{Si}(111)$ :As substrates with miscut along [112] lead to a significant twin reduction. We thus recommend to use substrates with such a miscut when growing GaP buffer layers on top of Si for optoelectronic and other applications that profit from low defect density. In contrast, a miscut along $[11 \overline{2}]$ assists twin formation due to the step configuration.

For the specific system of $\mathrm{GaP}$ on $\mathrm{Si}(111)$ :As, we found that the thermodynamically preferred interface configuration can essentially be considered as an interfacial GaAs layer between substrate $\mathrm{Si}: \mathrm{As}(111)$ and GaP epilayer. Whenever Si(111):As was miscut, we observed the formation of double bilayer steps in our experiments.

The mechanisms we have identified to reduce the defect density when nucleating GaP on As-modified Si(111) may prove useful for other hetero-systems as well, since twinning is a fundamental and general problem in (111)related epitaxy. We note that the central mechanism explained in this paper is based on bond counting near edges and is active also for single-stepped substrates. Our main results are the clarification that miscut substrates can yield epilayers having reproducibly and substantially fewer defects than flat surfaces and the discovery of the underlying mechanism to reduce the defect density.

We want to conclude with a list of a few more observations: Although more often used in a macroscopic context, the spreading factor remains a useful quantity to predict the growth type also on the microscopic level. The size distribution of the nuclei and the internal structure of the III-V layer can only be understood by distinguishing precisely between coherent and incoherent interfaces.

For similar setups where suppressed twinning is desired, the temperature should be sufficiently high to allow for precursor decomposition but otherwise as low as possible. Among other reasons, low temperature causes decreased surface mobility, which leads to smaller nuclei. Combined with a miscut substrate that yields substantially more untwinned nuclei, this results in small twinned nuclei that can then be overgrown easily. In the nucleation phase, the material supply should be very low in order to allow for internal reorganization (defect reduction) of the III-V material. The nucleation time has to be long enough to compensate for the low material supply: before growth starts, the substrate should be covered completely with III-V material. Further, high $\mathrm{V} / \mathrm{III}$ ratios were beneficial in our experiments. The experimental-theoretical findings presented here are meant to serve as a generic starting point for low-defect III-V epitaxy. 


\section{Appendix A: Appendix}

\section{DFT Parameters}

All DFT results were calculated using the plane wavecode VASP in version 5.4.171. The kinetic energy cutoff was set to be $600 \mathrm{eV}$. The Brillouin zone integration was performed with a $19 \times 19 \times 1(10 \times 10 \times 1)$ k-point Monkhorst-Pack mesh for the $1 \times 1(2 \times 2)$ slab calculations. Slab surfaces were separated by $12 \AA$ to $16 \AA$ vacuum. We employed the PBEsol density functional, which is optimized for solids. The atomic positions were fully relaxed using the conjugate gradient algorithm until all interatomic forces are smaller than $0.01 \mathrm{eV} / \mathrm{nm}$.

\section{DFT results for flat surface}

The configurations of Fig. 2 can be summarized in the following sequences of layer compositions (ordered from bulk to surface):

\begin{tabular}{c|ccccc}
\hline$I_{\alpha, \beta}$ & $\mathrm{Si}$ & $\mathrm{Ga}$ & $\mathrm{As}$ & $\mathrm{Ga}$ & $\mathrm{P}_{\alpha, \beta}$ \\
$I I_{\alpha, \beta}$ & $\mathrm{Si}$ & $\mathrm{Si}$ & $\mathrm{As}$ & $\mathrm{Ga}$ & $\mathrm{P}_{\alpha, \beta}$ \\
$I I_{\alpha, \beta}$ & $\mathrm{Si}$ & $\mathrm{Ga}_{0.75} \mathrm{Si}_{0.25}$ & $\mathrm{As}$ & $\mathrm{Ga}$ & $\mathrm{P}_{\alpha, \beta}$ \\
$I V_{\alpha, \beta}$ & $\mathrm{Si}$ & $\mathrm{Ga}_{0.75} \mathrm{Si}_{0.25}$ & $\mathrm{As}$ & $\mathrm{Ga}_{0.25} \mathrm{Si}_{0.75}$ & $\mathrm{P}_{\alpha, \beta}$ \\
\hline
\end{tabular}

In order to evaluate the size of the influence of lattice strain and hydrogen termination, we do the following comparison: Taking structure $I_{\alpha}$ as reference, i.e. $\Delta \gamma_{t o t}\left(I_{\alpha}\right)=0$, we obtain the following interface energies in $\mathrm{eV} / \mathrm{nm}^{2}$ from DFT calculations:

\begin{tabular}{c|cccc}
\hline$\Delta \gamma$ & $I_{\beta}$ & $I I_{\alpha}$ & $I I I_{\alpha}$ & $I V_{\alpha}$ \\
\hline$a_{S i}$ & 0.14 & 26.14 & 5.98 & 22.82 \\
$a_{m}$ & 0.17 & 26.27 & 6.03 & 23.08 \\
H-term & 0.05 & 34.89 & 8.50 & 34.74 \\
\hline
\end{tabular}

Here $a_{S i}, a_{m}$ and H-term denote systems fixed to the $\mathrm{Si}$ lattice constant, the average of the $\mathrm{Si}$ and $\mathrm{GaP}$ lattice constants, and a hydrogen-terminated system with $a_{m}$, respectively. Although the $\mathrm{Si}$ substrate presets the $\mathrm{Si}$ lattice constant, it might be realistic to assume that an average lattice constant will be realized near the interface. In the main text, we always refer to the results of the H-terminated case in order to avoid errors from dangling bonds. The numbers show that the numerical values are similar for all lattice constants and with/without hydrogen and hence the conclusions in the main text are independent of the particular choice.

We can further provide values for the energetic difference between the difference of the layer sequences $\mathrm{Si}-$ GaAs-GaP and Si-GaAs-PGa: The ordering Si-GaAs$\mathrm{GaP}$ is energetically preferred over $\mathrm{Si}-\mathrm{GaAs}-\mathrm{PGa}$ by about $3.17 \mathrm{eV} / \mathrm{nm}^{2}$, i.e. $0.42 \mathrm{eV}$ per atom $-0.411 \mathrm{eV}$ for $a_{m}$ and $435 \mathrm{meV}$ for $a_{S i}$. This is plausible, because III-V bonds are chemically preferred over $\mathrm{V}-\mathrm{V}$ bonds.

\section{DFT results for edge atoms}

Here, we compare binding energies obtained from DFT calculations for the $\alpha$ and $\beta$ configurations at doublesteps as shown in Fig. 5. The following table lists the difference in total energy between the double-steps reference structure without $\mathrm{P}$ and the same structure with one added $\mathrm{P}$ atom. All atomic positions have been relaxed until the interatomic forces were smaller than 0.01 $\mathrm{eV} / \mathrm{nm}$. The column "edge atom" refers to the coloredcoded purple atom in Fig. 5. First, we compare $\alpha$ and $\beta$ configurations on the lower level of the double-step. All numbers are in $\mathrm{eV}$, smaller (more negative) values correspond to higher cohesive energies.

\begin{tabular}{|c|c|c|c|c|}
\hline step & edge atom & $E_{\alpha}$ & $E_{\beta}$ & $\Delta E$ \\
\hline \multirow[t]{3}{*}{ DBBS } & $\mathrm{Ga}$ & -5.153 & -3.453 & +1.700 \\
\hline & $\mathrm{Si}$ & -6.768 & -6.342 & +0.426 \\
\hline & As & -5.996 & -5.900 & +0.096 \\
\hline \multirow[t]{3}{*}{ TBBS } & $\mathrm{Ga}$ & -3.893 & -5.663 & -1.769 \\
\hline & $\mathrm{Si}$ & -4.614 & -6.009 & -1.396 \\
\hline & As & -5.103 & -5.935 & -0.832 \\
\hline
\end{tabular}

We also compared $\alpha$ and $\beta$ positions for the upper $\mathrm{P}$ atom. In this case, we fixed As as lower edge atom.

\begin{tabular}{|c|c|c|c|c|}
\hline step & atom & $E_{\alpha}$ & $E_{\beta}$ & $\Delta E$ \\
\hline DBBS & As & -5.968 & -4.851 & +1.117 \\
\hline TBBS & As & -4.506 & -5.783 & -1.277 \\
\hline
\end{tabular}

Obviously, the energy difference is always positive (negative) for DBBS (TBBS), which means that $\alpha$ $(\beta)$ configurations are energetically preferred at DBBS (TBBS).

We further compare a row of Ga vs. a row of $\mathrm{P}$ as first atoms next to both DBBS and TBBS. The cohesive energy per atom is always higher for $\mathrm{P}$ than for Ga. Additionally to the fact that nucleation happens in a P-rich regime, this makes a nucleation starting with $\mathrm{P}$ adsorption a likely scenario (as described in the main text). For DBBS, the difference in binding energy per atom $(\mathrm{P}$ vs $\mathrm{Ga})$ is $2.07 \mathrm{eV}(\alpha)$ and $1.19 \mathrm{eV}(\beta)$ and for TBBS, 0.44 $\mathrm{eV}(\alpha)$ and $1.37 \mathrm{eV}(\beta)$ in favor of $\mathrm{P}$.

\section{Importance of kinetics for temperature dependence of $R_{\beta}$}

In order to put our model presented in the main text in perspective to other concepts, we briefly mention a few alternative theories that, however, are not compatible with our experimental data.

One could assume that the behavior of $R_{\beta}\left(T_{n u c}\right)$ is roughly described by canonical thermodynamics. Under the naive assumption that each atom takes one of the two possible states ( $\alpha$ or $\beta$ ) independently with a Boltzmann- 
factor, $p_{\alpha} \propto e^{-E_{\alpha} / k_{B} T_{n u c}}$, we could expect

$$
R_{\beta}=\frac{p_{\beta}}{p_{\alpha}+p_{\beta}}=\left(1+\exp \left[-\frac{E_{\alpha}-E_{\beta}}{k_{B} T_{n u c}}\right]\right)^{-1}
$$

However, this does not fit the data, e.g., the Arrheniuslike fit yields an effective activation energy of $858 \mathrm{meV}$ in the case of [112], whereas the single-atom energetic difference is below $50 \mathrm{meV}$.

Following a similar line of thoughts, one could as well that $R_{\beta}$ is determined by the ratio of clusters nucleating in $\beta$ configuration as described, e.g., by classical nucleation theory. For a critical free energy difference $\Delta G^{*}$ at a certain chemical potential $\mu$ this yields:

$$
R_{\beta}=\left(1+\exp \left[\frac{\Delta G^{*}}{k_{B} T_{n u c}}\right]\right)^{-1}
$$

However, there is no value $\Delta G^{*}$ for which this equation simultaneously yields $R_{\beta} \leq 0.1$ at $420^{\circ} \mathrm{C}$ and $R_{\beta} \geq 0.3$ at $500^{\circ} \mathrm{C}$, as required from the data. This is because small $R_{\beta} \leq 0.1$ result from large $\Delta G^{*}$ values in $\mathrm{A} 2$ which implies $\log R_{\beta} \cong \frac{-\Delta G^{*}}{k_{B} T_{n u c}}$. In an Arrhenius-like plot, this gives a straight line, which however goes through the origin - in strong contrast to the data from Fig. 4. Although classical nucleation theory has been successful applied to nanoscopic nuclei 29], it fails to predict twinning ratios in this case. A possible reason - strongly supported by the success of the $\mathrm{KMC}$ - is that the importance of kinetic aspects (like the temporal order of events) is underestimated.

As an alternative theory, one could argue that the diffusion length increases with temperature and hence, steps are more often hit by adsorbed particles. While this could explain the increase in $R_{\beta}$ for higher $T_{n u c}$ for miscut along [112], it fails to explain the corresponding increase in $R_{\beta}$ for miscut along [ [112], where the DBBS steps facilitate $\alpha$-configurations. Nucleation is likely to start at steps regardless of the specific temperature in the range from $420^{\circ} \mathrm{C}$ to $500^{\circ} \mathrm{C}$. Thus, the change in diffusion length alone cannot explain the experimental data.

\section{Stacking changes and domain boundaries}

We want to highlight that incoherent boundaries are not only seen between $\alpha$ and $\beta$ domains, but can also be present between two different $\beta$ domains if growth starts on neighboring step plateaus, cmp. incoherent stacking boundary in Fig. 11. This is illustrated in Fig. 10. It clearly shows that untwinned III-V material (e.g. GaP) can overgrow both step types (TBBS and DBBS) without introducing any defects (tension due to lattice mismatch is not considered here). Also no growth direction (step upwards or downwards) seems to be preferred. In the case of twinned nuclei, which are formed on neighboring plateaus and meet each other (independent from the exact position), a incoherent stacking boundary is formed (cf. Fig. 10 (c) and (d)). If untwinned III-V meets twinned nuclei the formation of an incoherent (lateral) $\Sigma 3$ twin boundary occurs. Here for both direction overgrowth is possible for every third layer, independent of their nucleation mechanism (cf. Fig. 10 (e) and (f)). Especially in compound semiconductors such incoherent defects are of high energy, due to charge compensation. The system prefers to avoid such defects by twin overgrowth and formation of coherent $\Sigma 3\{111 / 111\}$ boundaries. This results in a highly twinned zone close to the III-V/Si(111) interface, which is visible in several TEM investigations. 36, 72, 73] Interestingly it seems that in such twin|twin situations only overgrowth in one direction (step upwards) is possible, which might affect the resulting twin domain size.

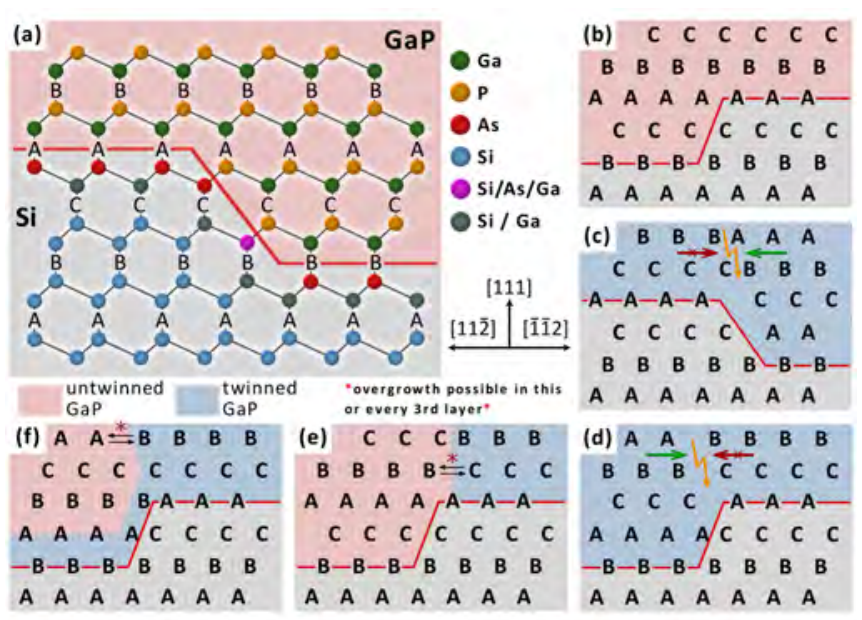

FIG. 10: Schematic side-view of $\mathrm{GaP} / \mathrm{Si}(111)$ :As along [110]: (a) shows the atomic lattice of untwinned GaP growth on Si(111) with DBBS. The drawn ABC-stacking follows the substrate without defects, which is also true for grown untwinned GaP over a TBBS (b). For simplicity, the following panels indicate only the ABC stacking, leaving out atoms and bonds. (c) and (d) show situations, where twinned GaP nucleates below and above the step-edge leading to the formation of a incoherent stacking (orange) somewhere near the step-edge. To avoid such energetically unfavored defects, the twins can overgrow each other by forming a coherent, low energy $\Sigma 3\{111 / 111\}$ boundary. The arrows (green + red) indicate that here such an overgrowth is only possible step upwards. Panel (e) shows untwinned GaP nucleating below the step-edge in contact with twinned GaP nucleating on the upper step plateau, while (f) represents a nucleation mechanism for twins at TBBS based on our model. In both cases the incoherent $\alpha \mid \beta$ twin-boundary is not fixed close to the step-edge. Every third layer, the stacking coincides again allowing for growth of one domain over another. 


\section{Dependence of $R_{\beta}$ on V/III ratio}

$\mathrm{GaP}$ growth is generally performed under an excess of the phosphorus precursor tertiarybutylphosphine (TBP) due to the higher desorption rate of $\mathrm{P}$ compared to Ga. In addition, TBP is self-limiting, i.e. $\mathrm{P}$ creates at most one monolayer on top of Ga. Gallium, on the other hand, tends to grow three-dimensional Ga clusters, which impedes 2D-growth of III-V epilayers in many cases. With higher V/III ratio, the Ga clustering can be suppressed, due to a reduced Ga diffusion. This results in smaller nuclei sizes as well as a higher nucleation density and thereby a faster layer coalescence. As discussed above and also claimed by Suzuki et al. [32], the volume-fraction of RTDs correlates with the nuclei size during the nucleation phase. Lower effective V/III ratios are indirectly realized as a side effect of lower nucleation temperature $T_{n u c}$, because the TBP decomposition is reduced at lower temperatures. Therefore, we want exclude the possibility that the change in twin ratio $R_{\beta}$ is merely such a indirect effect of $T_{n u c}$. To achieve that, we have prepared a series of samples with nominally identical conditions as those of Fig. 4 , but with different V/III pressure ratios. The results in Fig. 11 show that a reduction of $R_{\beta}$ with an increase of the V/III ratio. The magnitude of the effect is rather small and can not counteract the influence of $T_{n u c}$. This strengthens again the model in the main text and excludes the possibilty that the only relevant influence of $T_{n u c}$ on $R_{\beta}$ is due to a change in the $\mathrm{V} / \mathrm{III}$ ratio.

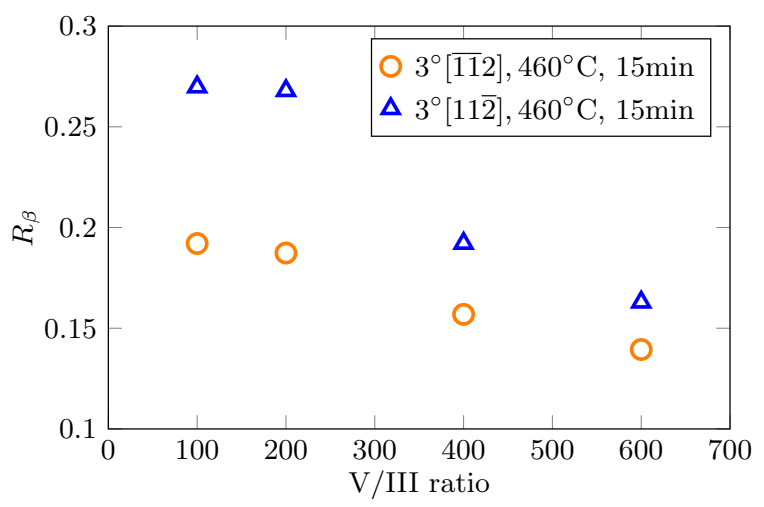

FIG. 11: The volume-ratio of twins $R_{\beta}$ decreases slightly with increasing V/III ratio. Although lower nucleation temperature $T_{n u c}$ reduces the P-supply and thereby the effective V/III ratio, the XRD-measured $R_{\beta}$ cannot be explained solely by such an indirect effect of changed temperature: Even in the wide range of deliberate six-fold increase in the V/III ratio (plotted here), we do not see such a strong change in $R_{\beta}$ as we observe by changing $T_{n u c}$, cmp. Fig. 7] Thus, $T_{n u c}$ must have an additional effect on the III- $V$ growth, which is mainly a kinetic one as described in section VIC

\section{Acknowledgments}

We would like to thank Oleksandr Romanyuk, Prague, for fruitful discussions. Special thanks are due to Ulli Bloeck, Helmholtz Zentrum Berlin, for TEM specimen preparation and analyses. We acknowledge financial support from the Thüringer Landesgraduiertenschule PhotoGrad.
[1] R. Chen, T.-T. D. Tran, K. W. Ng, W. S. Ko, L. C. Chuang, F. G. Sedgwick, and C. Chang-Hasnain,
Nature Photonics 5, 170 (2011), ISSN 1749-4885, 
1101.3305, URL http://www.nature.com/doifinder/ 10.1038/nphoton.2010.315

[2] D. Liang and J. E. Bowers, Nature Photonics 4, 511 (2010), ISSN 1749-4885, URL http://dx.doi.org/10. 1038/nphoton.2010.167http://linkinghub.elsevier. com/retrieve/pii/S0030399212003088http://www . nature.com/doifinder/10.1038/nphoton.2010.167

[3] K. Tomioka, M. Yoshimura, and T. Fukui, Nature 488, 189 (2012), ISSN 0028-0836, URL http://www.nature. com/doifinder/10.1038/nature11293

[4] H. Ko, K. Takei, R. Kapadia, S. Chuang, H. Fang, P. W. Leu, K. Ganapathi, E. Plis, H. S. Kim, S.-Y. Chen, et al., Nature 468, 286 (2011), ISSN 0028-0836, 1108.1127, URL http://www.nature.com/doifinder/10.1038/ nature09541http://arxiv.org/abs/1108.1127http: //dx.doi.org/10.1038/nature09541.

[5] T. Kawai, H. Yonezu, Y. Yamauchi, Y. Takano, and K. Pak, Applied Physics Letters 59, 2983 (1991), ISSN 0003-6951, URL http://aip.scitation.org/doi/10. 1063/1.105819

[6] R. S. Becker, J. A. Golovchenko, E. G. McRae, and B. S. Swartzentruber, Physical Review Letters 55, 2028 (1985), ISSN 00319007.

[7] A. Ohtake and K. Mitsuishi, Journal of Vacuum Science \& Technology B, Nanotechnology and Microelectronics: Materials, Processing, Measurement, and Phenomena 29, 031804 (2011), ISSN 2166-2746, URL http: //avs.scitation.org/doi/10.1116/1.3589807.

[8] Y. Ababou, P. Desjardins, A. Chennouf, R. Leonelli, D. Hetherington, A. Yelon, G. Lesperance, and R. A. Masut, Journal of Applied Physics 80, 4997 (1996), ISSN 0021-8979, URL http://aip.scitation.org/doi/ 10.1063/1.363544

[9] H. Yamaguchi, J. G. Belk, X. M. Zhang, J. L. Sudijono, M. R. Fahy, T. S. Jones, D. W. Pashley, and B. A. Joyce, Physical Review B 55, 1337 (1997), ISSN 0163-1829, URL http://link.aps.org/doi/10.1103/ PhysRevB.55.1337

[10] H. Yamaguchi, M. R. Fahy, and B. a. Joyce, Applied Physics Letters 69, 776 (1996), ISSN 0003-6951, URL http://aip.scitation.org/doi/10.1063/1.117888

[11] E. P. Kvam and R. Hull, Journal of Applied Physics 73, 7407 (1993), ISSN 0021-8979, URL http://aip. scitation.org/doi/10.1063/1.354033.

[12] I. Miccoli, P. Prete, F. Marzo, D. Cannoletta, and N. Lovergine, Crystal Research and Technology 46, 795 (2011), ISSN 02321300, URL http://doi.wiley.com/ 10.1002/crat. 201000711 .

[13] M. J. Evans, M. Stavola, M. G. Weinstein, and S. J. Uftring, Material Science and Engineering B 58, 118 (1999).

[14] M. Steger, A. Yang, T. Sekiguchi, K. Saeedi, M. L. W. Thewalt, M. O. Henry, K. Johnston, H. Riemann, N. V. Abrosimov, M. F. Churbanov, et al., Journal of Applied Physics 110, 081301 (2011), ISSN 00218979, URL http://scitation.aip.org/content/ aip/journal/jap/110/8/10.1063/1.3651774

[15] E. Bakkers, M. Borgstrom, and M. Verheijen, MRS Proceedings 1068, 1068 (2008), ISSN 19464274, URL http://journals.cambridge.org/ abstract\{_\}S1946427400028839.

[16] O. Supplie, O. Romanyuk, C. Koppka, M. Steidl, A. Nägelein, A. Paszuk, L. Winterfeld, A. Dobrich, P. Kleinschmidt, E. Runge, et al., Progress in Crystal
Growth and Characterization of Materials (2018), accepted Mai 2018.

[17] J.-H. Kang, Q. Gao, H. Joyce, H. Tan, C. Jagadish, Y. Kim, Y. Guo, H. Xu, J. Zou, M. Fickenscher, et al., in 2010 10th IEEE Conference on Nanotechnology, NANO 2010 (2010), ISBN 9781424470334.

[18] H. a. Fonseka, H. H. Tan, J. Wong-Leung, J. H. Kang, P. Parkinson, and C. Jagadish, Nanotechnology 24, 465602 (2013), ISSN 0957-4484, URL http://www.ncbi.nlm.nih.gov/pubmed/24157550http: //stacks . iop.org/0957-4484/24/i=46/a=465602?key= crossref.bdeabe485679bd2d657c6e4396314181

[19] S. G. Ghalamestani, S. Johansson, B. M. Borg, K. A. Dick, and L.-E. Wernersson, physica status solidi (c) 9, 206 (2012), ISSN 18626351, URL http://doi.wiley. com/10.1002/pssc. 201100258

[20] D. Salomon, A. Dussaigne, M. Lafossas, C. Durand, C. Bougerol, P. Ferret, and J. Eymery, Nanoscale research letters 8, 61 (2013), ISSN 1931-7573, URL http://www.pubmedcentral.nih. gov/articlerender.fcgi?artid=3576259\{\&\}tool= pmcentrez $\{\&\}$ rendertype=abstract $\{\%\} 5$ Cnhttp: //www.nanoscalereslett.com/content/8/1/61

[21] R. Songmuang, O. Landré, and B. Daudin, Applied Physics Letters 91 (2007), ISSN 00036951.

[22] H. Döscher, O. Supplie, S. Brückner, T. Hannappel, A. Beyer, J. Ohlmann, and K. Volz, Journal of Crystal Growth 315, 16 (2011), ISSN 00220248, URL http://linkinghub.elsevier.com/retrieve/pii/ S0022024810005464.

[23] O. Supplie, M. M. May, G. Steinbach, O. Romanyuk, F. Grosse, A. Nägelein, P. Kleinschmidt, S. Brückner, and T. Hannappel, The Journal of Physical Chemistry Letters 6, 464 (2015), ISSN 1948-7185, URL http:// pubs.acs.org/doi/abs/10.1021/jz502526e

[24] A. Paszuk, S. Brückner, M. Steidl, W. Zhao, A. Dobrich, O. Supplie, P. Kleinschmidt, W. Prost, and T. Hannappel, Applied Physics Letters 106, 231601 (2015), ISSN 0003-6951, URL http://dx.doi.org/10.1063/ 1.4922275http://scitation.aip.org/content/aip/ journal/apl/106/23/10.1063/1.4922275

[25] C. Koppka, A. Paszuk, M. Steidl, O. Supplie, P. Kleinschmidt, and T. Hannappel, Crystal Growth \& Design 16, 6208 (2016), URL http://dx.doi.org/10.1021/ acs.cgd.6b00541

[26] N. Nakagawa, H. Y. Hwang, and D. A. Muller, Nature Materials 5, 204 (2006), URL http://dx.doi.org/10. $1038 /$ nmat 1569 .

[27] K. Shimamura, Z. Yuan, F. Shimojo, and A. Nakano, Applied Physics Letters 103, 022105 (2013), ISSN 00036951, URL http://aip.scitation.org/doi/10.1063/ 1.4811746

[28] S. F. Fang, K. Adomi, S. Iyer, H. Morkoc, H. Zabel, C. Choi, and N. Otsuka, Journal of Applied Physics 68, R31 (1990), ISSN 00218979, URL http://scitation.aip.org/content/aip/journal/ jap/68/7/10.1063/1.346284.

[29] M. Steidl, C. Koppka, L. Winterfeld, K. Peh, B. Galiana, O. Supplie, P. Kleinschmidt, E. Runge, and T. Hannappel, ACS Nano 11, 8679 (2017), pMID: 28881138, http://dx.doi.org/10.1021/acsnano.7b01228, URL http: //dx.doi.org/10.1021/acsnano.7b01228

[30] A. Proessdorf, F. Grosse, O. Romanyuk, W. Braun, B. Jenichen, A. Trampert, and H. Riechert, Journal of 
Crystal Growth 323, 401 (2011), ISSN 00220248, URL http://dx.doi.org/10.1016/j.jcrysgro.2010.11. 167http://linkinghub.elsevier.com/retrieve/pii/ S0022024810011619.

[31] H. Suzuki, D. Ito, A. Fukuyama, and T. Ikari, Journal of Crystal Growth 380, 148 (2013), ISSN 00220248, URL http://dx.doi.org/10.1016/j.jcrysgro.2013. 06.015http://linkinghub.elsevier.com/retrieve/ $\mathrm{pii/S0022024813004193}$

[32] H. Suzuki, D. Ito, A. Haga, A. Fukuyama, and T. Ikari, in 2014 IEEE 40th Photovoltaic Specialist Conference (PVSC) (IEEE, 2014), 111, pp. 1830-1833, ISBN 978-1-4799-4398-2, URL http://ieeexplore.ieee.org/ document/6925279/.

[33] H. Suzuki, Y. Nakata, M. Takahasi, K. Ikeda, Y. Ohshita, O. Morohara, H. Geka, and Y. Moriyasu, AIP Advances 6, 035303 (2016), ISSN 2158-3226, URL http: //aip.scitation.org/doi/10.1063/1.4943511.

[34] Y. Kajikawa, Y. Son, H. Hayase, H. Ichiba, R. Mori, K. Ushirogouchi, and M. Irie, Journal of Crystal Growth (2016), ISSN 00220248, URL http://dx.doi.org/10. $1016 / \mathrm{j} \cdot$ jcrysgro.2016.12.062

[35] V. Narayanan, S. Mahajan, N. Sukidi, K. J. Bachmann, V. Woods, and N. Dietz, Philosophical Magazine A 80, 555 (2000), ISSN 0141-8610, URL http://www.tandfonline.com/doi/abs/10.1080/ 01418610008212068

[36] V. Narayanan, S. Mahajan, K. J. Bachmann, V. Woods, and N. Dietz, Philosophical Magazine A 82, 685 (2002), ISSN 0141-8610, URL http://www.tandfonline.com/doi/abs/10.1080/ 01418610208243196http://journalsonline.tandf .co. uk/Index/10.1080/01418610110082034.

[37] H. Kroemer, Journal of Crystal Growth 81, 193 (1987), ISSN 00220248, URL http://linkinghub.elsevier. com/retrieve/pii/0022024887903915

[38] T. Hannappel, W. McMahon, and J. Olson, Journal of Crystal Growth 272, 24 (2004), ISSN 00220248, URL http://linkinghub.elsevier.com/retrieve/ pii/S0022024804010267

[39] W. Zhao, M. Steidl, A. Paszuk, S. Brückner, A. Dobrich, O. Supplie, P. Kleinschmidt, and T. Hannappel, Applied Surface Science 392, 1043 (2017), ISSN 01694332, URL http://dx.doi.org/10.1016/j.apsusc.2016.09.081.

[40] O. Romanyuk, T. Hannappel, and F. Grosse, Physical Review B 88, 115312 (2013), ISSN 1098-0121, URL http://link.aps.org/doi/10.1103/PhysRevB.88. 115312 .

[41] J. E. Northrup, R. D. Bringans, R. I. G. Uhrberg, M. A. Olmstead, and R. Z. Bachrach, Physical Review Letters 61, 2957 (1988), ISSN 0031-9007, URL http://link. aps.org/doi/10.1103/PhysRevLett.61.2957

[42] M. A. Olmstead, R. D. Bringans, R. I. G. Uhrberg, and R. Z. Bachrach, Physical Review B 34, 6041 (1986), ISSN 0163-1829, URL http://link.aps.org/doi/10. 1103/PhysRevB.34.6041

[43] M. Copel, R. Tromp, and U. Kohler, Physical Review B 37, 10756 (1988).

[44] R. L. Headrick and W. R. Graham, Physical Review B 37, 1051 (1988), ISSN 0163-1829, URL http://link. aps.org/doi/10.1103/PhysRevB.37.1051.

[45] R. D. Bringans, R. I. G. Uhrberg, M. A. Olmstead, and R. Z. Bachrach, Physical Review B 34, 7447 (1986), ISSN 0163-1829, URL http://link.aps.org/doi/10.
1103/PhysRevB. 34.7447

[46] R. S. Becker, B. S. Swartzentruber, J. S. Vickers, M. S. Hybertsen, and S. G. Louie, Physical Review Letters 60, 116 (1988).

[47] J. R. Patel, J. A. Golovchenko, P. E. Freeland, and H. J. Gossmann, Physical Review B 36, 7715 (1987), ISSN 01631829 .

[48] M. D. Pashley, Physical Review B 40, 10481 (1989), URL https://link.aps.org/doi/10.1103/PhysRevB. 40.10481

[49] J. Wang, A. Misra, and J. P. Hirth, Physical Review B 83, 064106 (2011), ISSN 1098-0121, URL http://link. aps.org/doi/10.1103/PhysRevB.83.064106

[50] V. Kumar and B. S. R. Sastry, phys-

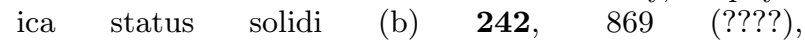
https://onlinelibrary.wiley.com/doi/pdf/10.1002/pssb.200402118, URL https://onlinelibrary.wiley.com/doi/abs/10. 1002/pssb. 200402118

[51] R. Roychowdhury, S. Kumar, A. Wadikar, C. Mukherjee, K. Rajiv, T. Sharma, and V. Dixit, Applied Surface Science 419, 957 (2017), ISSN 01694332, URL http://linkinghub.elsevier.com/retrieve/pii/ S0169433217313284

[52] S. Watanabe, K. Horiuchi, and T. Ito, Japanese Journal of Applied Physics 32, 3420 (1993), ISSN 0021-4922, URL http://stacks .iop.org/1347-4065/32/3420

[53] A. Antons, R. Berger, K. Schroeder, and B. Voigtländer, Physical Review B 73, 125327 (2006), ISSN 1098-0121, URL http://link.aps.org/doi/10.1103/PhysRevB. 73.125327

[54] R. J. Phaneuf, E. D. Williams, and N. C. Bartelt, Physical Review B 38, 1984 (1988), URL http://link.aps . org/doi/10.1103/PhysRevB.38.1984

[55] F. Patella, A. Sgarlata, F. Arciprete, S. Nufris, P. D. Szkutnik, E. Placidi, M. Fanfoni, N. Motta, and A. Balzarotti, Journal of Physics: Condensed Matter 16, S1503 (2004), ISSN 0953-8984, URL http://stacks.iop.org/0953-8984/16/i=17/a=005? key=crossref . cfb89323370f672ab531f0fbd0c50ede

[56] N. C. Bartelt, E. D. Williams, R. J. Phaneuf, Y. Yang, and S. Das Sarma, Journal of Vacuum Science \& Technology A: Vacuum, Surfaces, and Films 7, 1898 (1989), ISSN 0734-2101, URL http: //link. aip.org/link/?JVA/7/1898/1\{\&\}Agg=doihttp: //avs.scitation.org/doi/10.1116/1.576025

[57] A. V. Davydenko, A. G. Kozlov, and L. A. Chebotkevich, Journal of Applied Physics 116, 143901 (2014), ISSN 0021-8979, URL http://aip.scitation.org/doi/ 10.1063/1.4897536.

[58] J.-L. Lin, D. Y. Petrovykh, J. Viernow, F. K. Men, D. J. Seo, and F. J. Himpsel, Journal of Applied Physics 84, 255 (1998), ISSN 0021-8979, URL http://link.aip.org/link/?JAP/84/255/1http: //aip.scitation.org/doi/10.1063/1.368077.

[59] B. S. Swartzentruber, Y. Mo, M. B. Webb, and M. G. Lagally, Journal of Vacuum Science \& Technology A: Vacuum, Surfaces, and Films 7, 2901 (1989), ISSN 0734-2101, URL http://scitation.aip.org/content/ avs/journal/jvsta/7/4/10.1116/1.576167http: //avs.scitation.org/doi/10.1116/1.576167

[60] E. D. Williams and N. C. Bartelt, Science 251, 393 (1991), ISSN 0036-8075, URL http://www.sciencemag. org/cgi/doi/10.1126/science.251.4992.393.

[61] T. R. Ohno and E. D. Williams, Applied Physics Let- 
ters 55, 2628 (1989), ISSN 0003-6951, URL http://aip. scitation.org/doi/10.1063/1.101957.

[62] T. R. Ohno and E. Williams, Journal of Vacuum Science \& Technology B 8, 874 (1990), ISSN 0734211X.

[63] K. Schroeder, B. Engels, P. Richard, and S. Blügel, Physical Review Letters 80, 2873 (1998), ISSN 0031-9007, URL https://link.aps.org/doi/10.1103/ PhysRevLett.80.2873

[64] B. Voigtlander, A. Zinner, T. Weber, and H. P. Bonzel, Physical Review B 51 (1995).

[65] E. Kaxiras, Materials Science and Engineering: B 30, 175 (1995), ISSN 09215107, URL http://linkinghub. elsevier.com/retrieve/pii/0921510794090122.

[66] S. Ranganathan, Acta Crystallographica 21, 197 (1966), URL https ://doi.org/10.1107/S0365110X66002615

[67] S. Li, N. Buchan, C. Larsen, and G. Stringfellow, Journal of Crystal Growth 96, 906 (1989), ISSN 00220248, URL http://linkinghub.elsevier. com/retrieve/pii/0022024889906519

[68] S. Breuer, M. Hilse, A. Trampert, L. Geelhaar, and H. Riechert, Physical Review B 82, 075406 (2010), ISSN 1098-0121, URL https://link.aps.org/doi/10.1103/
PhysRevB.82.075406

[69] S. Gorji Ghalamestani, M. Berg, K. A. Dick, and L.-E. Wernersson, Journal of Crystal Growth 332, 12 (2011), ISSN 00220248, URL http://linkinghub.elsevier. com/retrieve/pii/S0022024811006014.

[70] D. K. Biegelsen, R. D. Bringans, J. E. Northrup, and L. E. Swartz, Applied Physics Letters 57, 2419 (1990), ISSN 0003-6951, URL http://aip.scitation.org/doi/ 10.1063/1.103864

[71] G. Kresse and J. Furthmüller, Physical Review B 54, 11169 (1996), URL https://link .aps .org/doi/10. 1103/PhysRevB.54.11169.

[72] H. Y. Xu, Y. N. Guo, Y. Wang, J. Zou, J. H. Kang, Q. Gao, H. H. Tan, and C. Jagadish, Journal of Applied Physics 106, 083514 (2009), ISSN 0021-8979, URL http: //aip.scitation.org/doi/10.1063/1.3248372

[73] K. Volz, A. Beyer, W. Witte, J. Ohlmann, I. Németh, B. Kunert, and W. Stolz, Journal of Crystal Growth 315, 37 (2011), ISSN 00220248, URL http://linkinghub. elsevier.com/retrieve/pii/S0022024810007669 